\title{
Grand Unified SU(8) Gauge Theory Based on Baryons which Are Yang-Mills Magnetic Monopoles
}

\author{
Jay R. Yablon \\ Schenectady, New York, USA \\ Email: jyablon@nycap.rr.com \\ Received January 15, 2013; revised April 22, 2013; accepted April 27, 2013
}

Copyright (C) 2013 Jay R. Yablon. This is an open access article distributed under the Creative Commons Attribution License, which permits unrestricted use, distribution, and reproduction in any medium, provided the original work is properly cited.

\begin{abstract}
Based on the thesis that baryons including protons and neutrons are Yang-Mills magnetic monopoles which the author has previously developed and which has been confirmed by over half a dozen empirically-accurate predictions, we develop a GUT that is rooted in the SU(4) subgroups for the proton/electron and neutron/neutrino which were used as the basis for these predictions. The SU(8) GUT group so-developed leads following three stages of symmetry breaking to all known phenomenology including a neutrino that behaves differently from other fermions, lepto-quark separation, replication of fermions into exactly three generations, the Cabibbo mixing of those generations, weak interactions which are left-chiral, and all four of the gravitational, strong, weak, and electromagnetic interactions. The next steps based on this development will be to calculate the masses and energies associated with the vacuum terms of the Lagrangian, to see if additional empirical confirmations can be achieved, especially for the proton and neutron and the fermion masses.
\end{abstract}

Keywords: GUT; SU(8); Yang-Mills; Baryons; Magnetic Monopoles; Nuclear Physics; Binding Energy; Protons; Neutrons; Fermions; Quarks; Electrons; Neutrinos; Leptons; Generations; Cabibbo Mixing; Chirality; Gravitation; QCD; Electroweak

\section{Introduction}

In a recent paper [1], the author introduced the thesis that baryons, including protons and neutrons, are Yang-Mills magnetic monopoles. Based on this thesis, it was possible to predict that the electron rest mass is related to the masses of the up and down quarks according to

$m_{e}=\left(m_{d}-m_{u}\right) /(2 \pi)^{\frac{3}{2}} \quad((11.22)$ of [1]), with the factor of $(2 \pi)^{\frac{3}{2}}$ emerging following a Gaussian integration over three space dimensions. Subsequent calculations showed that the best known values of the up and down masses in turn lead a binding energy of $7.667 \mathrm{MeV}$ per the proton and $9.691 \mathrm{MeV}$ per neutron yielding an average binding energy of $8.679 \mathrm{MeV}$ per nucleon ((12.6) through (12.8) of [1]), very much in accord with what is empirically observed, and to binding energies for ${ }^{56} \mathrm{Fe}$ which were predicted to be extremely close to what is observed for that nuclide. Noting also that the deuteron binding energy is extremely close to what is known from best available data to be the mass of the up quark, we further hypothesized that these might be one and the same, which could be explained if the energies released during nuclear fusion are based on some form of "resonant cavity" analysis in which the discreet energies which are observed to be released are based on the masses of the quarks contained within the nucleons and nuclides. This led to a prediction that ${ }^{56} \mathrm{Fe}$ has a latent available binding energy of $493.028394 \mathrm{MeV}((12.14)$ of [1]), which we then contrasted to the empirical binding energy of $492.253892 \mathrm{MeV}$. This small difference was understood as indicating that $99.8429093 \%$ of the available binding energy predicted by this model of nucleons as Yang-Mills magnetic monopoles goes into binding together the ${ }^{56} \mathrm{Fe}$ nucleus, and that the remaining $0.1570907 \%$ goes into confining the quarks within the nucleons. This in turn, lead us by the conclusion of [1] to a deepened understanding of how quark confinement is intimately related to nuclear binding and fission and fusion and the peak in per nucleon binding energies at ${ }^{56} \mathrm{Fe}$, and perhaps to an understanding of the so-called First ECM effect (see [1], pp. 62 and 66).

A second paper [2] extended this analysis, and showed that based on this same "resonant cavity" analysis, the binding energies of the remaining $1 \mathrm{~s}$ nuclides, namely ${ }^{3} \mathrm{H}$, ${ }^{3} \mathrm{He}$ and ${ }^{4} \mathrm{He}$, can be predicted to at least parts per hun- 
dred thousand and in most cases parts per million. This latter paper also showed that the observed neutron-proton mass difference is predicted by the relationship

$$
M(n)-M(p)=m_{u}-\left(3 m_{d}+2 \sqrt{m_{\mu} m_{d}}-3 m_{u}\right)(2 \pi)^{\frac{3}{2}}
$$

(in (7.2) of [2]) to better than 1 part per million. In Section 10 of [2], we explained why this should be regarded as an exact relationship, and therefore modified our earlier hypothesis that the deuteron binding energy is exactly equal to the up quark mass, into one in which these energies are very close-to just over 8 parts in ten million-but not exactly the same. In Section 9 of [2] we used these results to predict solar fusion energies solely from up and down quark masses, and found the results to also be in very tight accord with the observed data.

The lesson taken from [1,2] together, is that empirical evidence strongly supports the thesis that Yang-Mills magnetic monopoles are in fact baryons on the basis of seven independent predictions which closely match the experimental data, specifically: 1) the electron mass in relation to the up and down masses, 2) the ${ }^{56} \mathrm{Fe}$ binding energy specifically, and the per-nucleon binding energies on the order of $8.68 \mathrm{MeV}$ in general, 3) the proton minus neutron mass difference, and 4-7) the four distinct nuclide binding energies predicted for 4) $\left.\left.{ }^{2} \mathrm{H}, 5\right){ }^{3} \mathrm{H}, 6\right){ }^{3} \mathrm{He}$ and 7) ${ }^{4} \mathrm{He}$. The study of solar fusion in Section 9 of [2] does not contain anything independent of the predictions 1) through 7), but rather applies several of these predicttions in combination, and underscores that a "resonant cavity" analysis of nucleons and nuclides does consistently lead to empirically-accurate binding energies, evidenced by all of predictions 3) through 7) above.

While the theoretical foundation for all of these successful predictions was laid throughout [1], it was the field strength tensors for the proton and neutron, (11.3) and (11.4) of [1], reproduced below:

$$
\begin{aligned}
& \operatorname{Tr} F_{\mathrm{P}}^{\mu v} \\
& =-i\left(\frac{\bar{\psi}_{d}\left[\gamma^{\mu}{ }_{v} \gamma^{v}\right] \psi_{d}}{{ }_{\rho_{d}}-m_{d} "}+2 \frac{\bar{\psi}_{u}\left[\gamma^{\mu}{ }_{v} \gamma^{v}\right] \psi_{u}}{" \rho_{u}-m_{u} "}\right), \\
& \operatorname{Tr} F_{\mathrm{N}}^{\mu \nu} \\
& =-i\left(\frac{\bar{\psi}_{u}\left[\gamma^{\mu}{ }_{v} \gamma^{v}\right] \psi_{u}}{{ }_{\rho_{u}}-m_{u} "}+2 \frac{\bar{\psi}_{d}\left[\gamma^{\mu}{ }_{v} \gamma^{v}\right] \psi_{d}}{" \rho_{d}-m_{d} "}\right),
\end{aligned}
$$

when used to calculate the energy according to

$$
E=\frac{1}{2} \operatorname{Tr} \iiint F_{\mu v} F^{\mu v} \mathrm{~d}^{3} x
$$

((11.7) of [1]), which formed the specific basis for the calculations that led to all of these predictions. These field strength tensors, in turn, emerged as stable magnetic monopoles following specification of the $\mathrm{SU}(4)_{\mathrm{P}}$ "protium" and $\mathrm{SU}(4)_{\mathrm{N}}$ "neutrium" gauge groups in Section 7 of [1], followed by breaking the symmetry of these groups using the baryon minus lepton number generator $B-L$ via $\Phi=v_{G U T}(B-L) \quad((8.1)$ of [1]). So we take the thesis presented in Sections 7 and 8 of [1] that the protons and neutrons emerge following the $B-L$ breaking of the $\mathrm{SU}(4)_{\mathrm{P}}$ and $\mathrm{SU}(4)_{\mathrm{N}}$ groups to be supported by the compelling evidence of predictions 1) through 7), and so regard $\mathrm{SU}(4)_{\mathrm{P}}$ and $\mathrm{SU}(4)_{\mathrm{N}}$ as subgroups that do describe the real physical universe, not just some arbitrary groups that may or may not appear in the natural world. In short, we take accurate empirical predictions 1) through 7) above as direct evidence of the physical reality of SU(4) $)_{\mathrm{P}}$ and $\mathrm{SU}(4)_{\mathrm{N}}$.

Based on all of the foregoing, we shall in this paper take $\mathrm{SU}(4)_{\mathrm{P}}$ and $\mathrm{SU}(4)_{\mathrm{N}}$ as physically-validated, reliable building blocks for developing a "Grand Unified Theory" (GUT) based on the empirically-confirmed thesis that baryons, including protons and neutrons, are Yang-Mills magnetic monopoles.

\section{Unification and Grand Unification in Physical Science}

At least since the time when Isaac Newton hypothesized that the terrestrial "force" which caused an apple to fall from a tree was the same as the celestial "force" which guided the movements of the planets, unification has been a central objective of physical science. The preeminent scientist, entrepreneur and statesman Benjamin Franklin catapulted to fame when he realized that the terrestrial sparks he was creating in his laboratory were of a unified piece with the lightning from the heavens, and applied that understanding in a very practical way to develop lightning rods which cured an epidemic of mid18th century electrocutions throughout Europe brought about by the superstition of sending church bellringers to steeples at the highest place in town to clang large metallic bells to ward off the anger of the Gods every time a lightning storm approached. James Clerk Maxwell in 1873 elaborated what to that date was, and perhaps even to today's date is, the preeminent physical unification and at least the very paradigm of unification, as he pulled together the disparate threads of Gauss, Faraday and Ampere into a unifying set of equations for electricity and magnetism. This was deepened a generation later with Einstein and Minkowski's Lorentz-invariant unification of space and time. In these and similar endeavors the underlying theme has always been the same: to take what appear on their surface to be disparate natural phenomena, and acquire a deeper understanding which shows them to be governed by a single, common princeple. The success of past unifications leaves today's gen- 
eration of physicists with the firm conviction that further unifications can still be achieved, and that one day in the future, all of the laws of nature can and will be deduced from one common vantage point. After all, what is natural science other than an endeavor to explain what is observed through our direct senses and the clever instrumentation that extends our senses, by relating those observations to mathematically precise laws of nature which apply consistently, uniformly and replicably, without exception, in the broadest possible range of circumstances?

So-called "Grand Unified Theories," or GUTs, are part and parcel of this esteemed tradition, and are based specifically on the advent of Yang-Mills gauge theories and the realization that these Yang-Mills theories have a remarkable capacity to explain what is observed in nature as evidenced though their already-successful application to weak and strong and electroweak interactions. The Georgi-Glashow SU(5) model [3] which was reviewed at some length in Section 8 of [1] was one of the first "GUTs" and is perhaps the best known. The basic idea of Georgi-Glashow and any other GUT is to be able to represent all of the fermions which are observed in nature, and all of their interactions, using a single, simple gauge group with a symmetry which is then broken in one or more stages to arrive at the particle and interaction phenomenology observed in a laboratory setting. The fermions are the up and down quarks, the electron and neutrino leptons, and ideally their higher-generational carbon copies distinguished from the first generation solely by larger mass. The generators of the gauge group represent "interactions" of which there are understood to be four: gravitational, strong, weak and electromagnetic. The eigenvalues of the diagonalized generators of the gauge group, which are linearly related to discrete natural numbers such as $\frac{2}{3}$ and $-\frac{1}{3}$ and $\pm \frac{1}{2}$ and -1 and 0 , represent the "charges" of these fermions with respect to these interactions. A particular fermion may be associated with a particular eigenstate (eigenvector) of a representation of the GUT gauge group if all of its eigenvalues for all of the generators match up with what are known to be the charges of that fermion with respect to all of these interactions. So, for example, an electron is by definition the fermion eigenstate for which the lepton number eigenvalue $L=1$, the baryon number eigenvalue $B=0$, and the electric charge eigenvalue $Q=-1$. And the transitions/decays of a fermion from one eigenstate into another, or its interactions in a given eigenstate, lead to the mediating vector bosons of the theory. The trick in any GUT, is to characterize all of the interrelated charges of all of the fermions in the "simplest" way possible, to understand the stages and ways in which the symmetry of the group is broken starting at ultra-high energies and working down to energies which can be reached in a laboratory setting, and of course, to end up with something that accurately comports with all observed empirical data.

With this in mind, and as used in the discussion here, we distinguish "GUTs" from "unified field theories" more generally, as that subset of unified field theories which is specifically centered on understanding fermions and their interactions via their discrete charges using Yang-Mills gauge groups, and on making whatever observable predictions can be made based on such an understanding. So, for example, Kaluza-Klein theory, which to this day represents an exceedingly elegant classical unification of general relativity with Maxwell's electrodynamics using a fifth spacetime dimension that from today's vantage point is best understood as the "matter dimension" [4], is most certainly a form of "unified field theory" (and one which in the view of this author warrants more universal acceptance than it has at present, especially given that what we know of YangMills gauge theory should permit both gravitation and electromagnetism in Kaluza-Klein form to be extended into non-Abelian domains). But Kaluza-Klein is not a GUT in the sense that GUTs are focused on the use of Yang-Mills gauge groups to represent fermions and their interactions, and Kaluza Klein, at least absent a YangMills extension, has nothing to say about fermions. While one may define the term "GUT" more expansively to also include so-called "supersymmetric" theories, the foregoing defines by example, what we have in mind in this paper when referring to a "GUT", as opposed to a "unified field theory" without the GUT qualifier.

The Galilean foundation for all of modern science is that theory must be the confirmed by observation, and that the goal or at least an important by-product of theory is to systematically explain observation. For physical theorists, the pursuit is about systematically comprehending nature and confirming that comprehension based on experimental data, or as Hawking and Einstein have more loftily put it, "reading the mind of God." Because GUTs necessarily theorize about the behavior of nature at ultra-high energies such as $10^{15} \mathrm{GeV}$ and even higher that are unlikely to ever be reached by human experimentation under any foreseeable circumstances (with the possible exception of what we can learn by peering back billions of years through astronomical telescopes), such GUT theories necessarily opine on physics that may forever be beyond the reach of direct experimental confirmation. So the only way to discern the primacy of one GUT over another is indirectly, by virtue of what it predicts about low energy phenomenology that we can or may soon be able to observe. So as we consider how to construct the "puzzle" which is a GUT and decide what "pieces" to use in that puzzle, we want to start with puz- 
zle pieces that already are solidly-grounded in empirical observation.

Based on the seven independent predictions enumerated in the last section which closely match the empirical nuclear binding and related data based on the thesis that baryons are Yang-Mills magnetic monopoles, the GUT that we develop here will start with the $\mathrm{SU}(4)_{\mathrm{P}}$ and $\mathrm{SU}(4)_{\mathrm{N}}$ gauge groups developed in Sections 7 and 8 of [1], knowing that these groups now have been validated by over half a dozen independent pieces of empirical data from nuclear and particle physics. Additionally, because we have shown in [1,2] how to connect these gauge groups to energy numbers which can be and indeed have been empirically confirmed, an important objective in developing a GUT on the basis of $\mathrm{SU}(4)_{\mathrm{P}}$ and $\mathrm{SU}(4)_{\mathrm{N}}$ is to lay the foundation for perhaps obtaining additional, similar, successful predictions of other known energies which have been crying out for theoretical understanding for decades, most particularly, and most importantly, the free proton and neutron masses, and the observed fermion masses.

If it should be possible on the basis of a particular GUT to make accurate predictions of the proton and neutron and/or fermion masses, then even absent the ability to ever directly observe the $10^{15} \mathrm{GeV}$ and higher energy phenomena which lead to these predictions, such predicttions would certainly be solid evidence, albeit through indirect inference rather than direct observation, that such a GUT has also explained to us how nature behaves behind the veil of energies that we shall most certainly never get to directly observe (again, with possible astronomical caveat).

In other words, because a GUT, by its very nature, seeks to reach into energy domains that will likely be forever beyond human reach, it must fulfill the Galilean project by accurately explaining all of the masses and energies that we do observe through the instrumentation that does rest within our grasp, while at the same time teaching us about physics at energies that we shall likely never have the capacity to see directly. It is the prediction of the energies and masses we do observe, that gives us some measure of confidence that we are not being led astray by what the GUT tells us about the physics of unreachable energies. To use a different metaphor, GUTs seek to teach us about an entire iceberg, most of which we shall never be able to observe. So what the GUT teaches us about the tip of that iceberg which we can see, must be solidly-confirmed by empirical data every step of the way for us to have some confidence in what it teaches us about the rest of the iceberg which will forever remain out of sight.

Based on the foregoing, the purpose of this paper is to develop a GUT rooted in the thesis that baryons are Yang-Mills magnetic monopoles and the seven success- ful predictions which have already emanated from that thesis in [1,2], and to lay the foundation for additional mass and energy predictions, including those of the free proton and neutron and the fermion masses.

\section{Some Clues for Pursuing the Proton, Neutron and Fermion Masses}

Before we can make predictions of the proton and neutron and fermion masses, we must construct a reliable, empirically-grounded GUT, and we must know how to break its symmetry. Why do we say this?

We have already shown in [2] how the nuclear binding energies in the $1 \mathrm{~s}$ shell arise from using the field strength tensors (1.1) and (1.2) to calculate an energy

$E=-\iiint \mathfrak{L}_{\text {gauge }} \mathrm{d}^{3} x$ via the pure gauge terms in the Lagrangian (3.8) of [2]:

$$
\mathfrak{L}_{\text {binding }}=\frac{1}{2}\left((2 \pi)^{\frac{3}{2}} F_{A B} \cdot F_{B A}-F_{A A} \cdot F_{B B}\right),
$$

together with components $m_{u}, m_{d}$ and $\sqrt{m_{u} m_{d}}$ of the outer product $E_{P A B C D}((4.9)$ through (4.11) of [2]). But these binding energies are calculated using only the pure gauge field terms (3.1) of the Lagrangian developed in (3.12) of [2], written with the terms slightly regrouped:

$$
\begin{aligned}
\mathfrak{L}= & \frac{1}{2}\left((2 \pi)^{\frac{3}{2}} F_{\mu \nu A B} F_{B A}^{\mu v}-F_{\mu v A A} F_{B B}^{\mu v}\right) \\
& +(2 \pi)^{\frac{3}{2}}\left(D_{\mu} \Phi\right)_{A B}\left(D^{\mu} \Phi\right)_{B A}-\left(D_{\mu} \Phi\right)_{A A}\left(D^{\mu} \Phi\right)_{B B}(3.2) \\
& +(2 \pi)^{\frac{3}{2}} \mu^{2} \Phi_{A B} \Phi_{B A}-\mu^{2} \Phi_{A A} \Phi_{B B} \\
& +\frac{1}{2}(2 \pi)^{\frac{3}{2}} \lambda\left(\Phi_{A B} \Phi_{B A}\right)^{2}-\frac{1}{2} \lambda\left(\Phi_{A A} \Phi_{B B}\right)^{2} .
\end{aligned}
$$

We have not yet even begun to develop these other terms at all, yet it is made very clear by the development in $[1,2]$ that additional energy numbers can and will arise from complete development of these terms. So, we must develop these additional terms and we will look to them to perhaps lead us to the proton and neutron and fermion masses. But because all of these terms contain the vacuum $\Phi$, the actual numeric energy values we obtain from these $\Phi$-containing terms will depend upon the GUT gauge group we choose, and upon its vacua $\Phi$ and how these vacua are used to break symmetry. (We use the plural vacua because we have in mind breaking symmetry in sequence using the Planck vacuum on the order of $10^{19} \mathrm{GeV}$, so called GUT vacuum on the order of $10^{15} \mathrm{GeV}$, and the Fermi vacuum $v_{F}=246.219651$ $\mathrm{GeV}$ used to break electroweak interactions to electromagnetic interactions via $\left.S U(2)_{W} \times U(1)_{Y} \rightarrow U(1)_{E M}.\right)$ For example, given from (3.11) of [2] that: 


$$
\left(D_{\mu} \Phi\right)_{E F}=\partial_{\mu} \Phi_{E F}-i\left(\left[G_{\mu}, \Phi\right]\right)_{E F},
$$

we see that terms in (3.2) with $\left(D_{\mu} \Phi\right)\left(D^{\mu} \Phi\right)$ will mix gauge fields $G_{\mu}$ with vacuum fields $\Phi$. So whereas the pure gauge terms (3.1) led to expressions such as (4.9) and (4.10) of [2], namely:

$$
\begin{aligned}
& (2 \pi)^{\frac{3}{2}} E_{P A B C D}=\frac{1}{2}(2 \pi)^{\frac{3}{2}} \iiint F_{P A B} \cdot F_{P C D} \mathrm{~d}^{3} x \\
& =\left(\begin{array}{ccc}
\sqrt{m_{d}} & 0 & 0 \\
0 & \sqrt{m_{u}} & 0 \\
0 & 0 & \sqrt{m_{u}}
\end{array}\right) \otimes\left(\begin{array}{ccc}
\sqrt{m_{d}} & 0 & 0 \\
0 & \sqrt{m_{u}} & 0 \\
0 & 0 & \sqrt{m_{u}}
\end{array}\right), \\
& (2 \pi)^{\frac{3}{2}} E_{N A B C D}=\frac{1}{2}(2 \pi)^{\frac{3}{2}} \iiint F_{N A B} \cdot F_{N C D} \mathrm{~d}^{3} x \\
& =\left(\begin{array}{ccc}
\sqrt{m_{u}} & 0 & 0 \\
0 & \sqrt{m_{d}} & 0 \\
0 & 0 & \sqrt{m_{d}}
\end{array}\right) \otimes\left(\begin{array}{ccc}
\sqrt{m_{u}} & 0 & 0 \\
0 & \sqrt{m_{d}} & 0 \\
0 & 0 & \sqrt{m_{d}}
\end{array}\right),
\end{aligned}
$$

we should be alert to opportunities to develop mixed gauge field/vacuum terms where one of these matrices is replaced by a vev, especially the Fermi vev $v_{F}=$ $246.219651 \mathrm{GeV}$, so we can develop an energy "toolbox" with such expressions as $\sqrt{v_{F} \cdot m_{u}}$ and $\sqrt{v_{F} \cdot m_{d}}$. Why the Fermi vev? And why these square root expressions? Because numerical inspection of the square roots of the three main masses in (4.11) of [2] used to calculate binding energies throughout [2], times the square root of the Fermi vev, shows that:

$$
\begin{aligned}
& \sqrt{v \cdot m_{u}}=739.960397 \mathrm{MeV}, \\
& \sqrt{v \cdot m_{d}}=1099.12211 \mathrm{MeV}, \\
& \sqrt{v \cdot \sqrt{m_{u} m_{d}}}=901.835259 \mathrm{MeV} .
\end{aligned}
$$

These clearly are at exactly the right order of magnitude to explain the free proton and neutron masses $\mathrm{M}(\mathrm{p})$ $=938.272046(21) \mathrm{MeV}$ and $\mathrm{M}(\mathrm{n})=939.565379(21)$ $\mathrm{MeV}$, if and when we can put (3.6) through (3.8) and like expressions into the right context and obtain the right coefficients. And where do such coefficients come from? The generators of a GUT! (The author's subsequent paper in this same special issue of JMP starts with (3.8) to indeed successfully explain the free neutron and proton rest masses.)

So the proton and neutron masses, via the order of magnitude analysis above, straddle right down the middle of the Fermi vev and the masses of the quarks. One should therefore be on the lookout for ways to exploit this via the "mixed" gauge field $G_{\mu} /$ vacuum $\Phi$ terms in Lagrangian (3.2). And as noted at the end of Section 10 of [2], one should keep in mind that relation

$$
M(n)-M(p)=m_{u}-\left(3 m_{d}+2 \sqrt{m_{\mu} m_{d}}-3 m_{u}\right)(2 \pi)^{\frac{3}{2}}
$$

for the free neutron-proton mass difference now allows us to find the neutron and proton masses individually, so long as we can find sum expression which involves the sum of these masses. So it may well be that our target should be $M(n)+M(p)$ or some multiple thereof (perhaps the ${ }^{4} \mathrm{He}$ alpha nucleus studied extensively in [2]?) rather than either of these masses individually.

For another example, we go all the way back to (2.1) of [1], Maxwell's charge equation:

$$
\begin{aligned}
J^{v} & =\partial_{\mu} F^{\mu v}=\partial_{\mu} D\left[{ }^{\mu} G^{v}\right] \\
& =\partial_{\mu} \partial\left[{ }^{\mu} G^{v}\right]-i\left[G^{\mu}, G^{v}\right] \rightarrow J^{v} \\
& =\left(\partial_{\mu} \partial^{\mu}+m^{2}\right) G^{v}-\partial_{\mu} \partial^{v} G^{\mu}-i\left[G^{\mu}, G^{v}\right]
\end{aligned}
$$

with $D^{\mu} \equiv \partial^{\mu}-i G^{\mu}$, and where in the final term we have hand-added a "Proca mass." Based on (3.3), we can readily specify an analogous field equation:

$$
\begin{aligned}
& J \equiv \partial_{\mu} F^{\mu} \equiv \partial_{\mu} D^{\mu} \Phi=\partial_{\mu} \partial^{\mu} \Phi-i \partial_{\mu}\left[G^{\mu}, \Phi\right] \\
& \rightarrow J=\left(\partial_{\mu} \partial^{\mu}+m^{2}\right) \Phi-i \partial_{\mu}\left[G^{\mu}, \Phi\right]
\end{aligned}
$$

for a Yang-Mills (non-commuting) scalar field $\Phi$ with a scalar source $J$. In fact, this is just the Klein-Gordon equation for a non-Abelian (non-commuting) Yang-Mills scalar field with a non-zero scalar source, into which we have hand-added a Proca mass in the usual way. The reason this is of interest is that a central step in Section 2 of [1] was to develop the inverse $G_{v} \equiv I_{\sigma v} J^{\sigma}$ and then introduce fermion field wavefunctions $\psi$ via $J^{\mu}=\bar{\psi} \gamma^{\mu} \psi$, so that we went from $G_{v} \rightarrow I_{\sigma v} J^{\sigma} \rightarrow I_{\sigma \nu} \bar{\psi} \gamma^{\sigma} \psi$. But we can follow an analogous path with (3.10) by building scalar source $J$ out of fermions via $J \equiv \bar{\psi} \psi$. Then we can develop an inverse via $\Phi \equiv I J$ and follow the analogous progression $\Phi \rightarrow I J=\mathrm{I} \bar{\psi} \psi$. Consequently, the terms of the Lagrangian (3.2) quadratic in the scalar field can be developed as $\Phi \Phi \rightarrow \mathrm{I}^{2} J^{2}=\mathrm{I}^{2} \bar{\psi} \psi \bar{\psi} \psi$, and then we can follow the path of section 3 of [1] by employing spin sums $\Sigma u \bar{u}=N^{2}(p+m) /(\mathrm{E}+m)$, with the full progression

$$
\begin{aligned}
\Phi \Phi & \rightarrow \mathrm{I}^{2} J^{2} \rightarrow \mathrm{I}^{2} \bar{\psi} \psi \bar{\psi} \psi \\
& \rightarrow\left(N^{2} \mathrm{I}^{2} /(\mathrm{E}+m)\right) \times \bar{\psi}(\rho+m) \psi
\end{aligned}
$$

Then, if we pursue the same course of development as in [1] from start to finish, when we finally reach the counterpart of (11.19) of [1] and collapse the propagators so that interactions occur essentially at a point, we will end up with a Lagrangian term of the schematic form:

$$
\begin{aligned}
\mathfrak{L} & =\Phi \Phi \rightarrow \mathrm{I}^{2} J \rightarrow \mathrm{I}^{2} \bar{\psi} \psi \bar{\psi} \psi \\
& \rightarrow \frac{N^{2} \mathrm{I}^{2}}{m(\mathrm{E}+m)} \bar{\psi} \psi \equiv m_{f} \bar{\psi} \psi
\end{aligned}
$$

But this is the form of a Fermion mass term in a La- 
grangian, with the mass of the fermion specifically identified with $m_{f}=N^{2} \mathrm{I}^{2} / m(\mathrm{E}+m)$. Concurrently, the vev $v$ should also enter into this when we break symmetry with a generator $G$ by setting $\Phi=v G$. So this is a possible prescription, using the ФФ terms in (3.2), for revealing a fermion rest mass out a Lagrangian while preserving gauge symmetry and thus maintaining renormalizability!

But because the specifics of all of this center around the vacua $\Phi$, it becomes essential to have the right GUT gauge group, and to know how to break its symmetry in appropriate sequence. As noted above, to do this, we begin to develop a GUT gauge group using the $\mathrm{SU}(4)_{\mathrm{P}}$ and $\mathrm{SU}(4)_{\mathrm{N}}$ gauge groups developed in Sections 7 and 8 of [1], knowing that these groups now have been validated by over half a dozen independent pieces of empirical evidence from nuclear and particle physics. We build upon these empirically-validated puzzle pieces in the hope that this run of positive empirical predictions will continue with the masses and energies predicted by the terms in (3.2) which include the vacua $\Phi$.

\section{An Unbroken SU(8) GUT Group which Accommodates All Fermions and Left and Right-Chiral States, All Interactions, Three Generations, and an Idiosyncratic Neutrino, with Nothing Missing and Nothing Superfluous}

The proton and the neutron, of course, form an SU(2) weak isospin doublet $(p, n)$ with $I^{3}=\left(+\frac{1}{2},-\frac{1}{2}\right)$, respectively. But both the proton and the neutron are composite entities comprising three quarks, and as we have argued and indeed supported with empirical nuclear binding data, they are Yang-Mills magnetic monopoles. So really, the proton/neutron doublet is a doublet of triplets, $((d, u, u),(u, d, d))$. And the left-chiral weak isospin quantum numbers $I_{L}^{3}$ associated with this triplet doublet are $\left(\left(-\frac{1}{2}, \frac{1}{2}, \frac{1}{2}\right)\left(\frac{1}{2},-\frac{1}{2},-\frac{1}{2}\right)\right)$.

In Section 7 of [1], we demonstrated that at ultra-high GUT energies the proton was part of a larger gauge group which we dubbed the SU(4) $)_{\mathrm{P}}$ "protium" group which includes the proton and the electron, and that the neutron was similarly part of a larger gauge group we dubbed the SU(4) "neutrium" group which includes the neutron and the neutrino. As we then showed in Section 8 and specifically (8.1) of [1], these two groups are broken by a vacuum $\Phi=v_{G U T}(B-L)$ of a "baryon minus lepton number" generator $B-L \equiv-\sqrt{\frac{8}{3}} \lambda^{15}$ such that a proton triplet $d_{R}, u_{G}, u_{B}$ is separated from the electron for $\mathrm{SU}(4)_{\mathrm{P}}$ and a neutron triplet $u_{R}, d_{G}, d_{B}$ is separated from the neutron for $\mathrm{SU}(4)_{\mathrm{N}}$, and each triplet becomes part of a topologically-stable magnetic monopole. It was on the basis of these proton and neutron triplets broken out from SU(4), that we successfully rendered the seven predictions summarized in Section 1, and also correctly derived the fusion energy released during the solar fission cycle strictly as a function of the up, down and electron fermion masses. So these triplets and the $\mathrm{SU}(4)_{\mathrm{P}}$ and $\mathrm{SU}(4)_{\mathrm{N}}$ groups in which they are embedded would appear to be very solid puzzle pieces for constructing a larger GUT which is well-grounded empirically. That is exactly what we shall do here.

Normally, one works from the phenomenological gauge group $S U(3)_{C} \times S U(2)_{W} \times U(1)_{Y}$ and tries to find larger simple groups $G$ which embed all of these and their associated fermions. The SU(5) model of GeorgiGlashow [3] reviewed at some length in Section 8 of [1] is a paradigmatic example. Here, we shall start with $\mathrm{SU}(4)_{\mathrm{P}}$ and $\mathrm{SU}(4)_{\mathrm{N}}$ which we know lead to accurate binding energy predictions, and seek to construct a larger simple gauge group which includes these two groups, and which also encompasses the usual phenomenological gauge group $S U(3)_{C} \times S U(2)_{W} \times U(1)_{Y}$. The group we shall choose? $S U(8) \supset S U(4)_{N} \times S U(4)_{P}$. This is a larger group than $\mathrm{SU}(5)$, but as we shall see, it brings with it numerous benefits including 1) the ability to accommodate a non-zero neutrino mass and thus righthanded chiral neutrinos which are omitted from $\mathrm{SU}(5) ; 2)$ the ability to accommodate all flavors and colors of fermion, as well as protons and neutrons, all in the fundamental group representation $(\mathrm{SU}(5)$ splits the fermions into a fundamental 5 and a non-fundamental 10 representation while omitting the right-chiral neutrino); 3) the ability to accommodate different left and right-handed chiral projections with respect to weak hypercharge $Y$ and weak isospin $I^{3}$, for all fermions; 4) a solution, at long last, to the mystery of fermion replication into exactly three generations, and 5) interaction generators that may well be associated with gravitation based on the manner in which the elusive neutrino stands alone with respect to all other fermions by having an exceedingly tiny neutrino mass that is orders of magnitude smaller than the masses of the other fermions, and based on the ability to finally understand the origins of fermion generation replication.

We construct this $\mathrm{SU}(8)$ group by establishing a fundamental representation containing the fermion octuplet $\left(v,\left(u_{R}, d_{G}, d_{B}\right), e,\left(d_{R}, u_{G}, u_{B}\right)\right)$. The neutron triplet $\left(u_{R}, d_{G}, d_{B}\right)$ and proton triplet $\left(d_{R}, u_{G}, u_{B}\right)$ are designnated in separate parenthesis for visual emphasis, and as we can see, the neutrium group $\left(v, u_{R}, d_{G}, d_{B}\right)$ occupies the first four members of this octuplet and the protium group $\left(e, d_{R}, u_{G}, u_{B}\right)$ occupies the last four members. Of course, what really counts are the quantum numbers, 
so let's now turn to those.

In (7.1) of [1], we established that for the protium quadruplet, the electric charge generator could be specified by $Q \equiv B-L-\frac{2}{\sqrt{3}} \lambda^{8}=-\frac{2}{\sqrt{3}}\left(\sqrt{2} \lambda^{15}+\lambda^{8}\right)$. But in (7.3) of [1], we were required to use a different electric charge generator, namely $Q \equiv \frac{2}{\sqrt{3}} \lambda^{8}$ for the neutrium quadruplet. This if $\mathrm{OK}$ when the proton and electron are treated separately from the neutron and neutrino, and this was good enough to get us over half a dozen good binding energy predictions and other empirically-supported relationships. But once we put all these fermions together into one octuplet representation of a unifying group this is no longer $\mathrm{OK}$, and we need to define a new electric charge generator that works uniformly for all of the fermions in the group.

So let us now see exactly how we can put these two groups together and what this implies for the nature of the GUT that does so. SU(8) of course contains seven diagonalized $8 \times 8$ generator matrices, so rather than take up visual space with seven $8 \times 8$ matrices in which all but the diagonal elements are zero, let us construct this group using the tables below which convey the same information more compactly in an easier-to-follow form.

First, as just noted, the electric charge generator is

$$
Q \equiv B-L-\frac{2}{\sqrt{3}} \lambda^{8}=-\frac{2}{\sqrt{3}}\left(\sqrt{2} \lambda^{15}+\lambda^{8}\right)
$$

for $\mathrm{SU}(4)_{\mathrm{P}}$, while it is $Q \equiv \frac{2}{\sqrt{3}} \lambda^{8}$ for $\mathrm{SU}(4)_{\mathrm{N}}$. So if we lay out the eight fermions of this octuplet in a vertical column and show the three generators $\lambda^{15}, \lambda^{8}, \lambda^{3}$ of $\mathrm{SU}(4)$ in the first three columns (we actually show $B-L \equiv-\sqrt{\frac{8}{3}} \lambda^{15}$ which is merely a linear multiple of $\lambda^{15}$ ), and then show generators for electric charge $Q$, right-chiral weak hypercharge $Y_{R}=2 Q$, left chiral weak hypercharge $Y_{L}=B-L$, and right-chiral weak isospin $I_{R}^{3}=0$, which are all linear combinations of one or more of the three generators $\lambda^{15}, \lambda^{8}, \lambda^{3}$, we end up with Table 1 below.

In Table 1 below, we have segregated the $\mathrm{SU}(4)_{\mathrm{P}}$ and $\mathrm{SU}(4)_{\mathrm{N}}$ generators $\lambda^{15}=-\sqrt{\frac{3}{8}}(B-L), \lambda^{8}, \lambda^{3}$ from the remaining generators, so that we can clearly see that there are three linearly-independent degrees of freedom. The remaining generators for $Q, Y_{R}, Y_{L}$, are all linear combinations of the first three generators, and so provide no additional degrees of freedom, while $I_{R}^{3}=0$ can be trivially obtained from any other generator using the coefficient 0 . We shall wish, in the course of our analysis, to maintain a focus on the independent degrees of freedom. What makes the upper neutrium quadruplet not unified with the lower protium quadruplet is the fact, as mentioned above, that although all the other generators have the same form (i.e., are invariant) as between the

Table 1. Fermions and generators of $\mathrm{SU}(4)_{\mathrm{N}}$ and $\mathrm{SU}(4)_{\mathrm{P}}$.

\begin{tabular}{|c|c|c|c|c|c|c|c|}
\hline & \multicolumn{3}{|c|}{ Linearly Independent Degrees of Freedom } & \multicolumn{4}{|c|}{ Linear Combinations } \\
\hline & $B-L=-\sqrt{\frac{8}{3}} \lambda^{15}$ & $\lambda^{8}$ & $\lambda^{3}$ & $Q=\frac{2}{\sqrt{3}} \lambda^{8}$ & $Y_{R}=2 Q$ & $Y_{L}=B-L$ & $I_{R}^{3}$ \\
\hline$v$ & -1 & 0 & 0 & 0 & 0 & -1 & 0 \\
\hline$u_{R}$ & $\frac{1}{3}$ & $\frac{1}{\sqrt{3}}$ & 0 & $\frac{2}{3}$ & $\frac{4}{3}$ & $\frac{1}{3}$ & 0 \\
\hline$d_{G}$ & $\frac{1}{3}$ & $-\frac{1}{2 \sqrt{3}}$ & $\frac{1}{2}$ & $-\frac{1}{3}$ & $-\frac{2}{3}$ & $\frac{1}{3}$ & 0 \\
\hline$d_{B}$ & $\frac{1}{3}$ & $-\frac{1}{2 \sqrt{3}}$ & $-\frac{1}{2}$ & $-\frac{1}{3}$ & $-\frac{2}{3}$ & $\frac{1}{3}$ & 0 \\
\hline & $"$ & $"$ & $"$ & $Q=B-L-\frac{2}{\sqrt{3}} \lambda^{8}$ & $"$ & $"$ & $"$ \\
\hline$e$ & -1 & 0 & 0 & -1 & -2 & -1 & 0 \\
\hline$d_{R}$ & $\frac{1}{3}$ & $\frac{1}{\sqrt{3}}$ & 0 & $-\frac{1}{3}$ & $-\frac{2}{3}$ & $\frac{1}{3}$ & 0 \\
\hline$u_{G}$ & $\frac{1}{3}$ & $-\frac{1}{2 \sqrt{3}}$ & $\frac{1}{2}$ & $\frac{2}{3}$ & $\frac{4}{3}$ & $\frac{1}{3}$ & 0 \\
\hline$u_{B}$ & $\frac{1}{3}$ & $-\frac{1}{2 \sqrt{3}}$ & $-\frac{1}{2}$ & $\frac{2}{3}$ & $\frac{4}{3}$ & $\frac{1}{3}$ & 0 \\
\hline
\end{tabular}


upper and lower quadruplets as denoted by the "dittos", the electric charge generators are defined by different linear combinations. So electric charge $Q$ is not an invariant as between these two quadruplets. It is worth noting that for all of these fermions, $Y_{L}=B-L$, so $Y_{L}$ is not itself a linearly-independent generator from $\lambda^{15}$.

The one generator that we do not see explicitly represented in the above, of course, is the generator $I_{L}^{3}= \pm \frac{1}{2}$ of left-chiral weak interaction, and this is related very intimately to the different $Q$ generators as highlighted above. So, let us now a) introduce $I_{L}^{3}$ and b) use this $I_{L}^{3}$ in combination with $Y_{L}$ which happens to be equal in all cases to $B-L$, to specify $Q \equiv Y_{L} / 2+I_{L}^{3}$ as is ordinarily done in electroweak theory. Then, having $Q$ in hand, and given $I_{R}^{3} \equiv 0$, we may further specify $Y_{R} \equiv 2 Q$ if we insist on the chiral-invariant relationship $Q \equiv Y_{L} / 2+I_{L}^{3} \equiv Y_{R} / 2+I_{R}^{3}$.

So we now take Table 1 above, introduce all seven of the SU(8) diagonalized generators with the normalization $\operatorname{Tr}\left(\lambda_{i}^{2}\right)=\frac{1}{2}$, and specify suitable linear combinations of these. Then, we review not only how this accommodates the fermions and generators in Table $\mathbf{1}$ above, but also the new interaction generators that are introduced and their possible physical significance.

Aesthetically, is very simple and natural for the eight fundamental flavors and colors of fermion $\left(v, u_{R}, d_{G}, d_{B}, e, d_{R}, u_{G}, u_{B}\right)$ to each be made a member of the fundamental representation of $\mathrm{SU}(8)$. And, be- cause one does have eight fermions in nature (per generation), a natural question is, why not use $\mathrm{SU}(8)$ ? Sometimes, what appears to be the simplest approach really is the simplest approach, and leads to the best results, and we don't have to try to unnaturally "squish" eight fermions into a smaller group like SU(5) and then lose the right-chiral neutrino and split the representations.

In this regard, the question we shall explore largely throughout the rest of this paper-which is one of the reasons why one might not use $\mathrm{SU}(8)$ - is whether $\mathrm{SU}(8)$ is simply too large and can or ought to be made smaller. (We shall answer this question, "no"!) By "too large," we refer not to aesthetics, but to superfluity: does this group introduce any extra, superfluous particles or interactions which simply do not appear anywhere in the natural world. Put concisely, the underlying question is this: is $\mathrm{SU}(8)$ sufficient, and is everything in SU(8) necessary? Does it yield everything, and not one iota more? (We shall answer these questions, "yes"!)

Specifically, in going from two disjoint SU(4) groups in Table 1 to one unified SU(8) group in Table 2, we have gone from three independent generators $\lambda^{15}, \lambda^{8}, \lambda^{3}$ to seven. Out of these four new generators, we have left three of these, $\lambda^{63}, \lambda^{48}, \lambda^{35}$, in their "native" form without alteration, pending further exploration of these generators below. The fourth new generator, $\lambda^{24}$, we do not show explicitly. Rather, we use the degree of freedom provided by $\lambda^{24}$ to introduce the left-chiral weak isospin generator $I_{L}^{3}$, which we define as a linear combination of six of the seven "native" generators according to:

Table 2. Fermions and generators of SU(8).

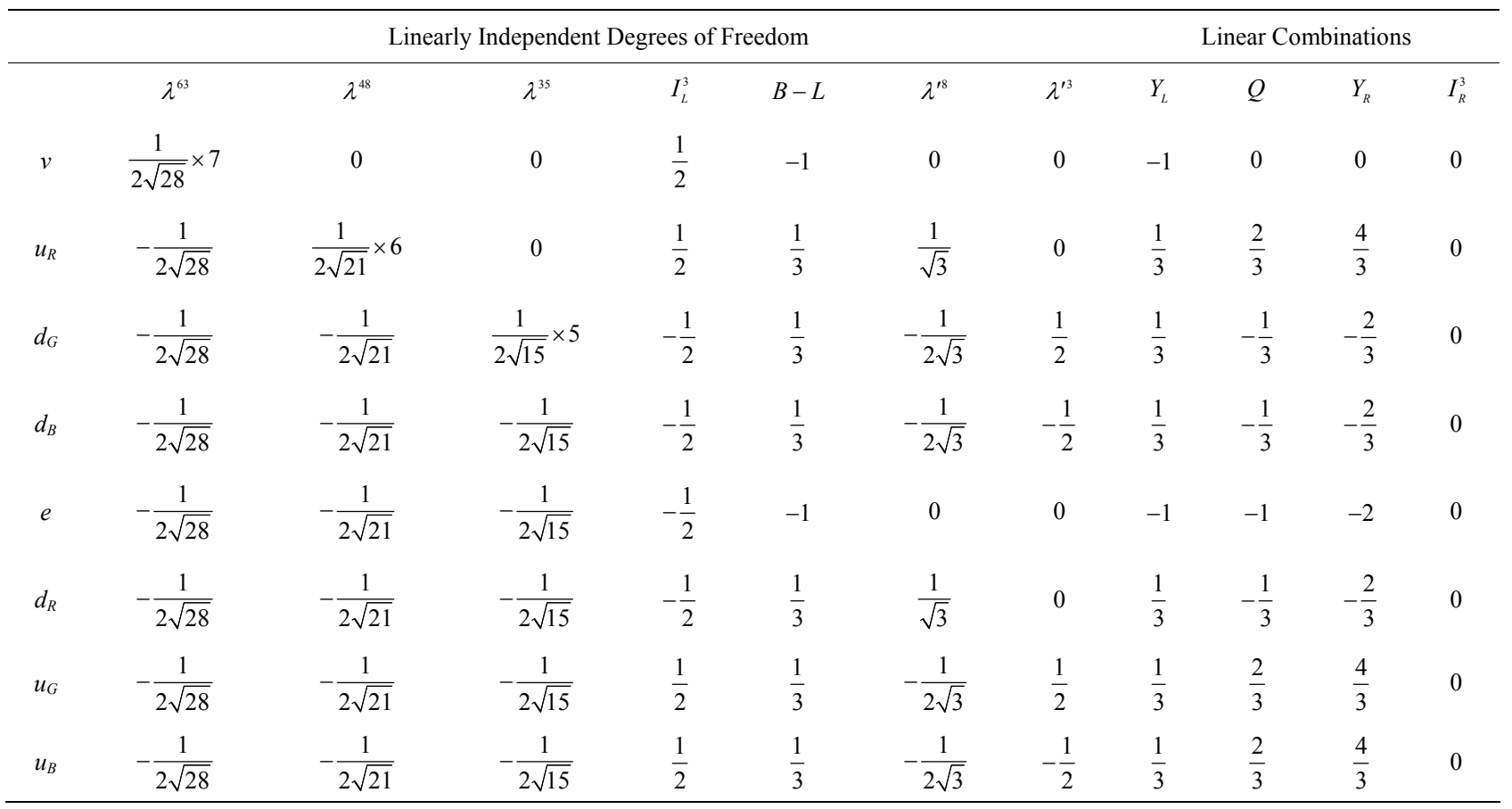




$$
\begin{aligned}
I_{L}^{3} & \equiv \frac{2}{\sqrt{7}} \lambda^{63}+\frac{4}{\sqrt{21}} \lambda^{48}-\frac{2}{\sqrt{15}} \lambda^{35} \\
& -\sqrt{\frac{2}{5}} \lambda^{24}-\sqrt{\frac{2}{3}} \lambda^{15}-\frac{2}{\sqrt{3}} \lambda^{8} .
\end{aligned}
$$

One can readily check that

$$
\operatorname{diag}\left(I_{L}^{3}\right)=\left(\frac{1}{2}, \frac{1}{2},-\frac{1}{2},-\frac{1}{2},-\frac{1}{2},-\frac{1}{2}, \frac{1}{2}, \frac{1}{2}\right)
$$

as in Table 2.

Now, for the bottom quadruplet with $\left(e, d_{R}, u_{G}, u_{B}\right)$, we have $B-L \equiv-2 \sqrt{\frac{2}{3}} \lambda^{15}$ as before. But this relationship needs to be replicated out of the native generators for the top quadruplet $\left(v, u_{R}, d_{G}, d_{B}\right)$ as well. This is realized by the following linear combination of native generators:

$$
\begin{aligned}
B-L \equiv & =-\frac{4}{\sqrt{7}} \lambda^{63}+\frac{4}{3 \sqrt{21}} \lambda^{48}+\frac{4}{9} \sqrt{\frac{3}{5}} \lambda^{35} \\
& +\frac{2}{3} \sqrt{\frac{2}{5}} \lambda^{24}-2 \sqrt{\frac{2}{3}} \lambda^{15}
\end{aligned}
$$

So we use (4.1) and (4.2) above to account for the two linearly-independent degrees of freedom in $\lambda^{24}$ and $\lambda^{15}$. It is easy to check as in Table 2 , that

$$
\operatorname{diag}(B-L)=\left(-1, \frac{1}{3}, \frac{1}{3}, \frac{1}{3},-1, \frac{1}{3}, \frac{1}{3}, \frac{1}{3}\right) .
$$

Similarly, we cannot use $\lambda^{8}$ alone, but must duplicate this as well for the top quadruplet $\left(v, u_{R}, d_{G}, d_{B}\right)$. This is achieved by defining a $\lambda^{\prime 8}$ generator:

$$
\lambda^{\prime 8} \equiv \frac{\sqrt{7}}{3} \lambda^{48}-\frac{2}{3 \sqrt{5}} \lambda^{35}-\sqrt{\frac{2}{15}} \lambda^{24}+\lambda^{8} .
$$

As required from Table 2, a check finds that

$$
\begin{aligned}
& \operatorname{diag}\left(\lambda^{\prime 8}\right) \\
& =\left(0, \frac{1}{\sqrt{3}},-\frac{1}{2 \sqrt{3}},-\frac{1}{2 \sqrt{3}}, 0, \frac{1}{\sqrt{3}},-\frac{1}{2 \sqrt{3}},-\frac{1}{2 \sqrt{3}}\right)
\end{aligned}
$$

Finally, and similarly, we need to define a $\lambda^{\prime 3}$ according to:

$$
\lambda^{\prime 3} \equiv \sqrt{\frac{3}{5}} \lambda^{35}-\sqrt{\frac{2}{5}} \lambda^{24}+\lambda^{3}
$$

This yields

$$
\operatorname{diag}\left(\lambda^{\prime 3}\right)=\left(0,0, \frac{1}{2},-\frac{1}{2}, 0,0, \frac{1}{2},-\frac{1}{2}\right)
$$

as in Table 2. The foregoing, (4.1) through (4.4), account for four of the seven linearly-independent degrees of freedom in $\mathrm{SU}(8)$. We have yet to explore the three native-form generators $\lambda^{63}, \lambda^{48}, \lambda^{35}$.
From here, we define several other generators which are linear combinations of (4.1) through (4.4). First, via (4.2), we define:

$$
\begin{aligned}
Y_{L} \equiv & B-L=-\frac{4}{\sqrt{7}} \lambda^{63}+\frac{4}{3 \sqrt{21}} \lambda^{48} \\
& +\frac{4}{9} \sqrt{\frac{3}{5}} \lambda^{35}+\frac{2}{3} \sqrt{\frac{2}{5}} \lambda^{24}-\sqrt{\frac{8}{3}} \lambda^{15}
\end{aligned}
$$

which happens to be exactly equal to $B-L$ in (4.2) and so is not linearly independent. But $Y_{L}$ is non-chiral, i.e., it only applies to left-chiral projections. Next, we use (4.5) and (4.1) to define the electric charge generator in the usual manner, via:

$$
\begin{aligned}
Q & \equiv \frac{1}{2} Y_{L}+I_{L}^{3}=\frac{1}{2}(B-L)+I_{L}^{3}+I_{R}^{3} \\
& =\frac{2}{3} \sqrt{\frac{7}{3}} \lambda^{48}-\frac{4}{3 \sqrt{15}} \lambda^{35}-\frac{2}{3} \sqrt{\frac{2}{5}} \lambda^{24} \\
& -2 \sqrt{\frac{2}{3}} \lambda^{15}-\frac{2}{\sqrt{3}} \lambda^{8}
\end{aligned}
$$

One can check to see that

$$
\operatorname{diag}(Q)=\left(0, \frac{2}{3},-\frac{1}{3},-\frac{1}{3},-1,-\frac{1}{3}, \frac{2}{3}, \frac{2}{3}\right),
$$

as required by Table 2 . In the third expression we make use of $I_{R}^{3}=0$, to show by way of contrast that Volovok's Equation (12.8) in [5] also leads via a different route to the exact same $Q=\frac{1}{2}(B-L)+I_{L}^{3}+I_{R}^{3}$.

Next, we formally specify that the right-chiral generator

$$
I_{R}^{3} \equiv 0
$$

is to be zero for all the fermions so that only left-chiral particles will interact weakly. At the same time we insist that the electric charge generator

$$
Q \equiv \frac{1}{2} Y_{L}+I_{L}^{3} \equiv \frac{1}{2} Y_{R}+I_{R}^{3}
$$

is to be defined as chiral symmetric for all fermions. This chiral insistence together with (4.6) and (4.7) finally leads to:

$$
\begin{aligned}
Y_{R}= & 2 Q=\frac{4}{3} \sqrt{\frac{7}{3}} \lambda^{48}-\frac{8}{3 \sqrt{15}} \lambda^{35}-\frac{4}{3} \sqrt{\frac{2}{5}} \lambda^{24} \\
& -4 \sqrt{\frac{2}{3}} \lambda^{15}-\frac{4}{\sqrt{3}} \lambda^{8}
\end{aligned}
$$

So at this point, all of the known quantum numbers of the fermions are fully specified, including the left and right chiral projections for $Y$ and $I^{3}$. The fermions all reside in the fundamental representation of $\mathrm{SU}(8)$, and the proton and neutron are represented as well in the way 
that we have ordered the fundamental representation. And, while all of the foregoing certainly accounts for the observed fermions and their quantum numbers, we still have three extra linearly-independent degrees of freedom, which we can and do choose to associate with the generators $\lambda^{63}, \lambda^{48}, \lambda^{35}$ we have left in their native state.

Now we return to the critical question: With these three apparently superfluous degrees of freedom, does $\mathrm{SU}(8)$ provide too much freedom? Does $\mathrm{SU}(8)$ provide more than what is necessary? Might we find some way, in the spirit of Georgi Glashow SU(5), to "squish" these fermions into a smaller group and take away some of this apparently-superfluous freedom? The answer is, no! And the reason is that this extra freedom is not superfluous, but is actually fully accounted for in the known particle phenomenology, and particularly, in the odd quirks of the neutrino and in the replication of fermion generations. Let us see how.

First, the neutrino. One of the very perplexing features of the neutrino is that it has almost no mass, and is maddeningly-elusive. While the electron and the quarks do have different masses from one another, the neutrinos are in a league of their own, by orders of magnitude. The neutrino mass is almost zero, which means that it travels at very close to the speed of light. Because of the equivalence of gravitational and inertial mass, the fact that the mass of the neutrino is so very different from that of all the other fermions means that in some rough manner of speaking, it is gravitating differently as well. For example, the mass of the electron's neutrino is less than 2 $\mathrm{eV}$ [6], while the electron itself has a mass of about 511 $\mathrm{KeV}$, which is over 250,000 times as large. This is of a totally different nature, involving completely different orders of magnitude, than $m_{u} / m_{e}=4.351853369$ and $m_{d} / m_{e}=9.601723351$ which are the relationships between the quark masses and the electron masses based on the quark masses arrived at in (10.3) and (10.4) of [2]. This appears to make the neutrino qualitatively different from all the other fermions, and we need to pinpoint the origins of this difference.

Now consider the $\lambda^{63}$ in Table 2 and the fact that $\lambda^{63}=-\frac{1}{4 \sqrt{7}}$ for all of the up and down quarks and the electron, but that $\lambda^{63}=+\frac{7}{4 \sqrt{7}}$ has a completely different value for the neutrino. Moreover, not only is the magnitude different by 7 to 1 , but even more importantly, the sign is different. Indeed, that is why we chose to place the neutrino as the very top member of the SU(8) fermion octuplet. That means that the neutrino will interact completely differently under the interaction associated with $\lambda^{63}$-whatever that interaction may befrom any other fermion. But if there is any interaction under which the neutrino behaves differently than all the other fermions, it is the gravitational interaction, because the most pronounced way in which the neutrino differs from the other fermions is via its ghostly mass and thus its ghostly way of gravitating. Further, we know on general principles that for any Yang-Mills gauge group which unifies gravitation with the other three interactions, there will have to be at least one degree of freedom given to the gravitational interaction. The only question is where and how this appears.

So, we now make a preliminary association of the $\lambda^{63}$ generator with a degree of freedom for a gravitational interaction, and we do so in a way that bakes in for the neutrino, an entirely different way of gravitating and thus displaying its mass, than any other fermion.

So, now we have accounted at least in a general way (which we shall seek to deepen in the upcoming discussion) for all four of the known interactions, but we still have two more degrees of freedom unaccounted for, namely, those provided by $\lambda^{48}, \lambda^{35}$. What are we to make of these? This brings us again to the question: does this not give us too much freedom? And again, the answer is, no!

We still have to account for the replication of fermions into three generations, which is another oddity of the material world almost as mysterious as the oddities of the neutrino just discussed. Let's ask the question directly: even if $\lambda^{63}$ is related to gravitation and can explain why the neutrino behaves so differently from all the other fermions, $\lambda^{48}, \lambda^{35}$ still give us two apparently superfluous degrees of freedom. What does this mean? What can we do with those extra two degrees of freedom? And specifically, might they be origin of generation replication?

Any time we have two degrees of freedom such as are provided by $\lambda^{48}, \lambda^{35}$, it is possible to construct three eigenstates out of those degrees of freedom. So, let us do just that, and label these states $e, \mu, \tau$ as in Figure 1 below.

We use "primes" in these generators, because if they do represent degrees of freedom associated with generation replication, they do not act in same way as the remaining generators $\lambda^{63}, \lambda^{24}, \lambda^{15}, \lambda^{8}, \lambda^{3}$ in Table 2 . These other five generators represent a "vertical" symmetry wherein each of the eight fermions is distinguished from one another by different quantum numbers. But the appearance of three generations in which the quantum numbers are identical from one generation to the next, and in which for a given fermion, generation is distinguished exclusively by rest mass, is a horizontal symmetry. And it is for and to this horizontal symmetry, that we shall develop and devote the generators $\lambda^{\prime 48}, \lambda^{\prime 35}$.

Now, in the forthcoming discussion, we shall seek to uncover in detail, the particular mechanism by which 
these two generators $\lambda^{48}, \lambda^{35}$ separate themselves from the remaining vertical generators $\lambda^{63}, I^{3}, B-L, \lambda^{\prime 8}, \lambda^{\prime 3}$ to situate themselves horizontally. The only point being made at the moment, is that two extra generators in Yang-Mills theory, such as $\lambda^{48}, \lambda^{35}$, provide enough freedom to support three distinct states as in Figure 1. And these three states will come equipped with their own $3 \times 3$ unitary matrices $U$ to mix these states. And, if we are asking ourselves whether the extra two generators $\lambda^{48}, \lambda^{35}$ provide too much freedom at the same time that we are seeking an explanation of the three fermion generations, and given that those two extra generators provide precisely the freedom needed to allow each particle to exist in one of three additional horizontal generational states, then perhaps these are not superfluous after all, but are instead the source of the generations. In that case, $\mathrm{SU}(8)$ becomes a perfect fit, large enough to accommodate all that is observed including the idiosyncratic behavior of the neutrino and the replication of fermion generations, and not one bit larger so as to contain anything superfluous that is not observed.

So in Table 3 below, we shall use the schematic symbol $\therefore$ to denote a visual shorthand for Figure 1 below: a condensed symbol that represents two degrees of freedom which are used to provide three distinct states $e, \mu, \tau$ which appear in Figure 1. And, let us replace the generators $\lambda^{48}, \lambda^{35}$ with this schematic to represent the horizontal symmetry of generation replication. Thus, we now rewrite Table 2 in the form of Table 3 as shown below.

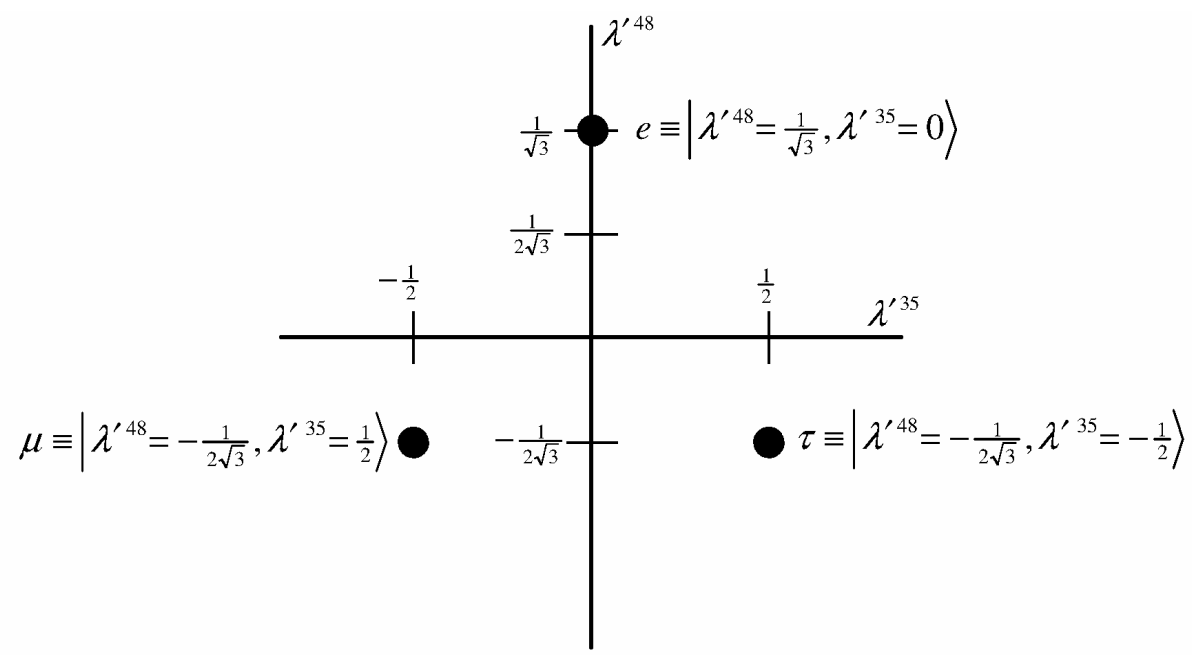

Figure 1. Three generation eigenstates constructed from $\lambda^{\prime 48}$ and $\lambda^{\prime 35}$.

Table 3. Fermions and generators of $\mathrm{SU}(8)$, with generation replication.

\begin{tabular}{|c|c|c|c|c|c|c|c|c|c|c|}
\hline & \multicolumn{6}{|c|}{ Linearly Independent Degrees of Freedom } & \multicolumn{4}{|c|}{ Linear Combinations } \\
\hline & $\lambda^{63}$ & $\lambda^{48}, \lambda^{35}$ & $I_{L}^{3}$ & $B-L$ & $\lambda^{\prime 8}$ & $\lambda^{\prime 3}$ & $Y_{L}$ & $Q$ & $Y_{R}$ & $I_{R}^{3}$ \\
\hline$v$ & $\frac{1}{2 \sqrt{28}} \times 7$ & $\therefore$ & $\frac{1}{2}$ & -1 & 0 & 0 & -1 & 0 & 0 & 0 \\
\hline$u_{R}$ & $-\frac{1}{2 \sqrt{28}}$ & $\therefore$ & $\frac{1}{2}$ & $\frac{1}{3}$ & $\frac{1}{\sqrt{3}}$ & 0 & $\frac{1}{3}$ & $\frac{2}{3}$ & $\frac{4}{3}$ & 0 \\
\hline$d_{G}$ & $-\frac{1}{2 \sqrt{28}}$ & $\therefore$ & $-\frac{1}{2}$ & $\frac{1}{3}$ & $-\frac{1}{2 \sqrt{3}}$ & $\frac{1}{2}$ & $\frac{1}{3}$ & $-\frac{1}{3}$ & $-\frac{2}{3}$ & 0 \\
\hline$d_{B}$ & $-\frac{1}{2 \sqrt{28}}$ & $\therefore$ & $-\frac{1}{2}$ & $\frac{1}{3}$ & $-\frac{1}{2 \sqrt{3}}$ & $-\frac{1}{2}$ & $\frac{1}{3}$ & $-\frac{1}{3}$ & $-\frac{2}{3}$ & 0 \\
\hline$e$ & $-\frac{1}{2 \sqrt{28}}$ & $\therefore$ & $-\frac{1}{2}$ & -1 & 0 & 0 & -1 & -1 & -2 & 0 \\
\hline$d_{R}$ & $-\frac{1}{2 \sqrt{28}}$ & $\therefore$ & $-\frac{1}{2}$ & $\frac{1}{3}$ & $\frac{1}{\sqrt{3}}$ & 0 & $\frac{1}{3}$ & $-\frac{1}{3}$ & $-\frac{2}{3}$ & 0 \\
\hline$u_{G}$ & $-\frac{1}{2 \sqrt{28}}$ & $\therefore$ & $\frac{1}{2}$ & $\frac{1}{3}$ & $-\frac{1}{2 \sqrt{3}}$ & $\frac{1}{2}$ & $\frac{1}{3}$ & $\frac{2}{3}$ & $\frac{4}{3}$ & 0 \\
\hline$u_{B}$ & $-\frac{1}{2 \sqrt{28}}$ & $\therefore$ & $\frac{1}{2}$ & $\frac{1}{3}$ & $-\frac{1}{2 \sqrt{3}}$ & $-\frac{1}{2}$ & $\frac{1}{3}$ & $\frac{2}{3}$ & $\frac{4}{3}$ & 0 \\
\hline
\end{tabular}


Now, in Table 3, SU(8) has nothing superfluous, all eight fermions are represented with both left and right-chiral states, and each can exist in one of the three $\therefore \equiv e, \mu, \tau$ horizontal generation eigenstates. We see that there are now four vertical interactions: 1) the strong QCD interaction with three color states and two generator degrees of freedom $\left.\lambda^{\prime 8}, \lambda^{\prime 3} ; 2\right)$ the weak isospin interaction represented by $I_{L}^{3}$;3) a $B-L$ interaction to which the electromagnetic interaction of (4.6) is linearly related by $Q \equiv \frac{1}{2} Y_{L}+I_{L}^{3}=\frac{1}{2}(B-L)+I_{L}^{3}$; and 4$)$ a final $\lambda^{63}$ providing a degree of freedom for a gravitational interaction, under which all fermions except the neutrino interact in one way, and under which the neutrino acts in a very different way, in a league by itself. This is the unbroken GUT group that seems best situated to fully accommodate not only all the known fermions and interactions and their key phenomenological properties, but the Yang-Mills magnetic monopoles which we now know are baryons, and which are very naturally grouped in this way of representing $\mathrm{SU}(8)$.

\section{Spontaneous Symmetry Breaking of SU(8) at the Planck and GUT Energy Scales, and the Emergence of Fermion Generations and Fermion Mass Degrees of Freedom}

In Section 8 of [1], we reviewed spontaneous symmetry breaking in the Georgi-Glashow SU(5) model, to provide a backdrop for breaking the protium group via $S U(4)_{P} \rightarrow S U(3)_{P C^{\prime}} \times U(1)_{B-L}$ and the neutrium group via $S U(4)_{N} \rightarrow S U(3)_{N C^{\prime}} \times U(1)_{B-L}$. This of course led to stable protons and neutrons and later to the several accurate empirical binding energy predictions already noted. Here, we review a similar symmetry breaking based on the $\mathrm{SU}(8)$ group developed in the previous section. Specifically, we review three symmetry breaking operations: a first symmetry breaking operation using the contemplated "gravitational" generator $\lambda^{63}$ at or near the Planck scale; a second symmetry breaking operation using the $Y_{L} \equiv B-L$ generator at an ultra-high GUT energy perhaps in the $10^{15} \mathrm{GeV}$ vicinity, and a third break of the electroweak symmetry at the Fermi scale using the electric charge generator $Q$. It is this third symmetry breaking that we hope to use to accurately predict the proton and neutron masses as discussed in Section 3 and highlighted in (3.6) to (3.8). But to set the context, let us start with the first two high-energy symmetry breaking operations using $\lambda^{63}$ and $Y_{L} \equiv B-L$.

If $\lambda^{63}$ is indeed a gravitational generator, then its mass scale will be at or near (within an order of magnitude of) the Planck mass which is defined by $G M_{P}^{2} \equiv \hbar c$, where $G$ is the gravitational constant and $\hbar c$ contains the Planck constant and the speed of light. In terms of energies that we have been discussing here,

$M_{P} \cong 1.221 \times 10^{19} \mathrm{GeV}$ is nineteen orders of magnitude larger than the proton mass. It is theorized that at this energy, there is a violent sea of vacuum perturbations, and two of the best references to review this understanding are $[7,8]$. We shall examine all of this more closely here as well, in the next section.

Without yet going through all the details in this pass, if we employ the Lagrangian (3.2) and specify a Planck vacuum $\Phi_{P}=\lambda^{i} \varphi_{P i} ; i=1, \cdots, 63$, we may break symmetry at or near $v_{P}=M_{P}$ using the $\lambda^{63}$ generator such that:

$$
\begin{aligned}
\Phi_{P} & \cong v_{P} \lambda^{63}, \text { i.e. }, \operatorname{diag}\left(\Phi_{P}\right)=\operatorname{diag}\left(\lambda^{i} \varphi_{P i}\right) \\
& \cong \frac{1}{2 \sqrt{28}} v_{P}(7,-1,-1,,-1,-1,-1,-1,-1)
\end{aligned}
$$

with $\varphi_{P i}=0 ; i=1, \cdots, 62$ otherwise. (Again, we are not concerned here with the exact relationship which why we use $\cong$ rather than $=$, but rather an order of magnitude examination of the qualitative features of this symmetry breaking.) This would immediately set the neutrino which is the top member of the elementary fermion octuplet $\left(v, u_{R}, d_{G}, d_{B}, e, d_{R}, u_{G}, u_{B}\right)$ on a course to behave differently from all the other particles. If $\lambda^{63}$ is indeed a gravitational degree of freedom which notion we began to entertain in the last section, then it makes sense to regard the degree of freedom that $\lambda^{63}$ provides to be a freedom associated with the rest mass of the fermion, i.e., to be a vertical mass degree of freedom. So with symmetry breaking of the neutrino from all the other fermions at the Planck scale, right below the Planck scale all of the fermions except the neutrino would have one mass, and the neutrino would have a different mass. Most notably, the neutrino would have an oppositely-signed generator from all of the other seven fermions, which we shall revisit in the next section. Thus, the neutrino can be expected right from the start, to behave very uniquely as regards its mass, and as regards to how it gravitates. This could be a root cause of why the quark mass to electron mass ratios are

$$
m_{u} / m_{e}=4.351853369, m_{d} / m_{e}=9.601723351,
$$

while $m_{e} / m_{v}>250,000$. One can envision that masses which are equal at the Planck scale might separate so that they differ from one another by factors of 4.35 to 1 or 9.60 to 1 at observable energies. But for a ratio $m_{e} / m_{v}>250,000$ we expect this to be more than just "screening adjustments" as we go from high to low energies. We expect this to be "baked in" to the underlying structure of the GUT gauge group right from the start.

Moving on, we now venture down to the vicinity of a second $v_{G U T} \cong 10^{15} \mathrm{GeV}$, where we break the symmetry 
with $Y_{L} \equiv B-L$. Again, we are simply for the moment talking about orders of magnitude for this energy scale. In fact, we have already discussed $B-L$ symmetry breaking at some length in Section 8 of [1]. But in that earlier discussion, we regarded $S U(4)_{P}$ and $S U(4)_{N}$ as disjoint groups each breaking down via

$$
\begin{aligned}
& S U(4)_{P} \rightarrow S U(3)_{P C^{\prime}} \times U(1)_{B-L}, \\
& S U(4)_{N} \rightarrow S U(3)_{N C^{\prime}} \times U(1)_{B-L}
\end{aligned}
$$

to produce a

$$
\pi_{1}\left(S U(3)_{C^{\prime}}\right) \times \pi_{1}\left(U(1)_{B}\right)=\pi_{1}\left(U(1)_{B}\right)=\mathbb{Z}
$$

homotopy group with stable magnetic monopoles, essentially based on the disjointed groups of Table 1. Now, in contrast, we have conjoined these groups into $\mathrm{SU}(8)$ as represented by Table 2 above. So the symmetry breaking we are about to explore is a "wholesale" breaking of $S U(4)_{P}$ and $S U(4)_{N}$ together at once in $\mathrm{SU}(8)$, versus the parallel, but "retail" symmetry breaking of $S U(4)_{P}$ and $S U(4)_{N}$ conducted in Section 8 of [1].

It is also worth noting as reviewed in Section 8 of [1], that Georgi and Glashow also break symmetry using the $Y$ generator, albeit such that

$$
\operatorname{diag}(\Phi)=\operatorname{diag}\left(T^{i} \varphi_{i}\right)=v_{G U T}\left(-\frac{1}{3},-\frac{1}{3},-\frac{1}{3}, \frac{1}{2}, \frac{1}{2}\right)
$$

for a right-chiral quintuplet $\left(d_{R}, d_{G}, d_{B}, e_{C},-v_{C}\right)_{R}$ of fermions. So here, we are doing exact same thing as Georgi and Glashow insofar as using a $Y$ generator to break the GUT symmetry circa $10^{15} \mathrm{GeV}$, but we are merely using a different group SU(8) versus SU(5), with all the fermions in the fundamental representation as shown in Table 2. Now let's proceed.

The group is now SU(8). Exactly as in (8.1) of [1], the vacuum we use is:

$$
\Phi_{G U T}=v_{G U T}(B-L)=v_{G U T} Y_{L}
$$

Here, however, because of the SU(8) group, we have:

$$
\begin{aligned}
& \operatorname{diag}\left(\Phi_{G U T}\right) \\
= & \operatorname{diag}\left(T^{i} \varphi_{i}\right) \equiv v_{G U T}\left(-1, \frac{1}{3}, \frac{1}{3}, \frac{1}{3},-1, \frac{1}{3}, \frac{1}{3}, \frac{1}{3}\right) \\
= & v_{G U T} \operatorname{diag}(B-L)=v_{G U T} \operatorname{diag} Y_{\mathrm{L}}
\end{aligned}
$$

Unlike Section 8 of [1], we no longer have

$B-L \equiv-\sqrt{\frac{8}{3}} \lambda^{15}$ from which we set $\varphi_{15}=-2 \sqrt{\frac{2}{3}} v_{G U T}$ and so obtain the Clebsch-Gordon coefficient via

$$
\varphi_{15}^{2}=\frac{8}{3} v_{G U T}^{2}=C^{2} v_{G U T}^{2} .
$$

Rather, here we have a $B-L$ specified in (4.2) which is a linear combination of five generators. Thus, to break symmetry here, picking off the coefficients in (4.2), we now must set:

$$
\begin{aligned}
& \varphi_{63}=-\frac{4}{\sqrt{7}} v_{G U T} ; \varphi_{48}=\frac{4}{3 \sqrt{21}} v_{G U T} ; \varphi_{35}=\frac{4}{9} \sqrt{\frac{3}{5}} v_{G U T} ; \\
& \varphi_{24}=\frac{2}{3} \sqrt{\frac{2}{5}} v_{G U T} ; \varphi_{15}=-2 \sqrt{\frac{2}{3}} v_{G U T}
\end{aligned}
$$

with all the remaining $\varphi_{i}=0$. The invariant scalar:

$$
\begin{aligned}
& \varphi_{63}^{2}+\varphi_{48}^{2}+\varphi_{35}^{2}+\varphi_{24}^{2}+\varphi_{15}^{2} \\
& =\left(\frac{16}{7}+\frac{16}{9 \times 21}+\frac{16 \times 3}{81 \times 5}+\frac{4 \times 2}{9 \times 5}+\frac{4 \times 2}{3}\right) v_{G U T}^{2} \\
& =\frac{80}{21} v_{G U T}^{2} \equiv C^{2} v_{G U T}^{2}
\end{aligned}
$$

yields a Clebsch-Gordon coefficient $C^{2}=\frac{80}{21}$ (Note the $C^{2}=\frac{8}{3}$ from the earlier $B-L \equiv-\sqrt{\frac{8}{3}} \lambda^{15}$ included in the calculation of the above). One may then employ the procedure such as is outlined in (11.5) and (11.6) of [1] to obtain gauge bosons masses in the usual way, and these will have masses on the order of $v_{G U T}$.

But our interest here is in what happens at lower energies, after this symmetry has been broken, because that brings us into energy ranges with are experimentally observable.

First, by breaking symmetry via

$$
\Phi=v_{G U T}(B-L)=v_{G U T} Y_{L},
$$

which for which the generator eigenvalues are

$$
\left(-1, \frac{1}{3}, \frac{1}{3}, \frac{1}{3},-1, \frac{1}{3}, \frac{1}{3}, \frac{1}{3}\right),
$$

we "fracture" the eight fermions in Tables $\mathbf{2}$ and $\mathbf{3}$ into a $Y_{L}=B-L=-1$ hypercharge doublet of leptons $(v, e)$ and a $Y_{L}=B-L=\frac{1}{3}$ hypercharge sextuplet of quarks $\left(\left(d_{R}, u_{G}, u_{B}\right)\left(u_{R}, d_{G}, d_{B}\right)\right)$. Of course, we know that $\left(d_{R}, u_{G}, u_{B}\right)$ is a proton and $\left(u_{R}, d_{G}, d_{B}\right)$ is a neutron, so this sextuplet may also be viewed as a $Y_{L}=B-L=1$ proton/neutron doublet $(p, n)$. Referring to Tables 2 and $\mathbf{3}$, the weak isospin for each doublet $(v, e),(p, n)$ is given by $I^{3}=\left(\frac{1}{2},-\frac{1}{2}\right)$. Of course for the proton this is arrived at by adding $I^{3}=\frac{1}{2}=-\frac{1}{2}+\frac{1}{2}+\frac{1}{2}$ for its three quarks, and for the neutron similarly via

$$
I^{3}=-\frac{1}{2}=\frac{1}{2}-\frac{1}{2}-\frac{1}{2} .
$$


Note also that by virtue of how the triplets in $\left(\left(d_{R}, u_{G}, u_{B}\right)\left(u_{R}, d_{G}, d_{B}\right)\right)$ are ordered, each entry in $\left(d_{R}, u_{G}, u_{B}\right)$ forms a weak isospin doublet with respect to its corresponding same-colored entry in $\left(u_{R}, d_{G}, d_{B}\right)$. Each of the three quarks also enjoys two color degrees of freedom $R, G, B$ associated with the $S U(3)_{C}$ ' generators $\lambda^{\prime 8}, \lambda^{\prime 3}$, see (4.3) and (4.4). So the group arrived at following $B-L$ symmetry breaking is schematically represented by:

$$
\begin{aligned}
& S U(8) \rightarrow S U(6)_{B} \times S U(2)_{L} \\
& =S U(3)_{C^{\prime}} \times S U(2)_{W} \times U(1)_{Y_{L}=B-L}
\end{aligned}
$$

The $\Phi=v_{G U T}(B-L)=v_{G U T} Y_{L} \quad$ symmetry breaking has fractured the quarks from the leptons into a sextuplet of quarks each with $B=\frac{1}{3}$ and a doublet of leptons each with $L=1$. Just as in Georgi/Glashow, this breaks a lepto-quark symmetry. This is the origin of the $S U(6)_{B} \times S U(2)_{L}$ factor. But the quarks are grouped into a proton and neutron doublet with $I^{3}=\left(\frac{1}{2},-\frac{1}{2}\right)$, and of course the two members of the lepton doublet also both have $I^{3}=\left(\frac{1}{2},-\frac{1}{2}\right)$. This is the well-known "isospin redundancy" that exists and between quarks/baryons and leptons and leads some to consider "preon" models such as that discussed in Section 12 of [5]. For quarks/baryons, we use $S U(3)_{C^{\prime}} \times S U(2)_{W} \times(1)_{Y_{L}=B-L}$ to represent their status following $Y_{L}=B-L$ symmetry breaking. That is, the proton and neutron each containing an $S U(3)_{C^{\prime}}$ color triplet of quarks, form an $S U(2)_{W}$ weak doublet $(p, n) \leftrightarrow S U(3)_{C^{\prime}} \times S U(2)_{W} \times(1)_{Y_{L}=B-L}$ with every single fermion containing an identical

$Y_{L}=B-L=\frac{1}{3}$, hence the $U(1)_{Y_{L}=B-L}$ factor. For leptons, the neutrino and electron form an $S U(2)_{W}$ weak doublet $(v, e) \leftrightarrow S U(2)_{W} \times(1)_{Y_{L}=B-L}$ with each containing an identical $Y_{L}=B-L=-1$, hence the $U(1)_{Y_{L}=B-L}$ factor, albeit for a different value of $Y_{L}=B-L$ than that of the quarks/baryons. Overall, with the detailed interrelationships just noted, we reproduce the phenomenological product group $S U(3)_{C} \times S U(2)_{W} \times U(1)_{Y}$.

Given that we have used $\Phi=v_{G U T}(B-L)=v_{G U T} Y_{L}$ for symmetry breaking at $v_{G U T}$, all that we have just described should be readily apparent from Tables 2 and 3. But a bonus that we obtain here, which is not obtained in Georgi-Glashow SU(5), is the fermion generation replication. This is how:

In $\mathrm{SU}(5)$ which is broken using $\Phi=v_{G U T} Y$, there are four degrees of freedom based on the linearly-independent generators $T^{24}, T^{15}, T^{8}, T^{3}$. After symmetry break- ing there are still four degrees of freedom; they are merelyreshuffled into $\lambda^{8}, \lambda^{3}$ for $S U(3)_{C}, I^{3}$ for $S U(2)_{W}$, and $Y$ for $U(1)_{Y}$. None of these degrees of freedom disappear after symmetry breaking; they simply sit across one another in several "irregular" linear combinations.

Here, however, in going from

$$
S U(8) \rightarrow S U(6)_{B} \times S U(2)_{L},
$$

two "vertical" degrees of freedom "disappear", because SU(8) has seven diagonalized generators while SU(6) has only five, and the separate $B$ and $L$ subscripts in $S U(8) \rightarrow S U(6)_{B} \times S U(2)_{L}$ are all part of a single degree of freedom represented by $Y_{L}=B-L$. But this reduction-by-two in the degrees of freedom cannot vanish into thin air; it must show up in some other way. That is, following symmetry breaking using $\Phi=v_{G U T}(B-L)=v_{G U T} Y_{L}$, there are two-free floating degrees of freedom from $\lambda^{48}, \lambda^{35}$ that have become decoupled from the remaining five vertical degrees of freedom. But, as shown in Figure 1, these free-floating degrees of freedom have precisely the properties needed to create a new horizontal freedom with exactly three states. So we label these three states $e, \mu, \tau$ as in Figure $\mathbf{1}$, we associate this with the fermion generation replication, and we therefore make a carbon copy of each fermion in triplicate, using the conventional symbols $u, d, c, s, t, b$ for the quarks, $e, \mu, \tau$ for the electrons, and $v_{e}, v_{\mu}, v_{\tau}$ for the neutrinos. The vertical quantum numbers associated with each type of fermion $u, c, t$; $d, s, b ; e, \mu, \tau$ and $v_{e}, v_{\mu}, v_{\tau}$ are identical for each triplet. The fermions across generations are distinguished only by the mass values, and so apparently, it is the freefloating generators $\lambda^{48}, \lambda^{35}$ which provide the horizontal fermion mass degrees of freedom to enable each fermion of a given type to take on one of three mass values. Thus we may formulate Table 4 below.

Studying Table 4 and the above comments about the generational mass freedom, we now can better develop our understanding of the so-called gravitational degree of freedom $\lambda^{63}$ which we discussed a short while ago in relation to (5.1). Whereas $\lambda^{48}, \lambda^{35}$ provide freedom for the fermions of any given type to take on one of three mass values, we also need a degree of freedom for each of the four basic fermion "prototypes" $v, e, u, d$ to have different masses within a single generation, as is also clearly observed. This, in fact, is the role of $\lambda^{63}$. While the neutrino is set on a different mass trajectory at the outset at the Planck scale because its $\lambda^{63}$ generator eigenvalue is $\frac{1}{2 \sqrt{28}} \times 7$ while that for all of the other fermions is the oppositely signed $-\frac{1}{2 \sqrt{28}}$ with $1 / 7$ the 
Table 4. Quarks and leptons with generation replication following $B-L=Y_{L}$ GUT symmetry breaking.

\begin{tabular}{|c|c|c|c|c|c|c|c|c|c|}
\hline & \multicolumn{5}{|c|}{ Linearly Independent Degrees of Freedom } & \multicolumn{4}{|c|}{ Linear Combinations } \\
\hline & $\lambda^{63}$ & $I_{L}^{3}$ & $B-L$ & $\lambda^{\prime 8}$ & $\lambda^{\prime 3}$ & $Y_{L}$ & $Q$ & $Y_{R}$ & $I_{R}^{3}$ \\
\hline \multicolumn{10}{|c|}{ Quarks } \\
\hline \multirow{6}{*}{$n\left\{\begin{array}{l}(u, c, t)_{R} \\
(d, s, b)_{G} \\
(d, s, b)_{B}\end{array}\right)$} & 1 & 1 & 1 & 1 & 0 & 1 & $\underline{2}$ & $\underline{4}$ & 0 \\
\hline & $2 \sqrt{28}$ & $\overline{2}$ & 3 & $\sqrt{3}$ & 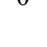 & $\overline{3}$ & $\overline{3}$ & $\overline{3}$ & 0 \\
\hline & 1 & 1 & 1 & 1 & 1 & 1 & 1 & 2 & 0 \\
\hline & $2 \sqrt{28}$ & $\overline{2}$ & $\overline{3}$ & $2 \sqrt{3}$ & $\overline{2}$ & $\overline{3}$ & $\overline{3}$ & $\overline{3}$ & 0 \\
\hline & 1 & 1 & 1 & 1 & 1 & 1 & 1 & 2 & 0 \\
\hline & $2 \sqrt{28}$ & $\overline{2}$ & $\overline{3}$ & $2 \sqrt{3}$ & $\overline{2}$ & $\overline{3}$ & $\overline{3}$ & $\overline{3}$ & \\
\hline \multirow{6}{*}{$p\left\{\left(\begin{array}{l}(u, c, t)_{G} \\
(u, c, t)_{B}\end{array}\right)\right.$} & 1 & -1 & $\underline{1}$ & 1 & 0 & $\underline{1}$ & -1 & -2 & 0 \\
\hline & $2 \sqrt{28}$ & 2 & 3 & $\sqrt{3}$ & & 3 & 3 & 3 & \\
\hline & 1 & 1 & 1 & 1 & 1 & 1 & 2 & 4 & o \\
\hline & $2 \sqrt{28}$ & $\overline{2}$ & $\overline{3}$ & $2 \sqrt{3}$ & $\overline{2}$ & $\overline{3}$ & $\overline{3}$ & $\overline{3}$ & 0 \\
\hline & 1 & 1 & 1 & 1 & -1 & 1 & $\underline{2}$ & $\underline{4}$ & 0 \\
\hline & $2 \sqrt{28}$ & $\overline{2}$ & $\overline{3}$ & $2 \sqrt{3}$ & $\overline{2}$ & $\overline{3}$ & $\overline{3}$ & $\overline{3}$ & 0 \\
\hline \multicolumn{10}{|c|}{ Leptons } \\
\hline$\left(v_{e}, v_{\mu}, v_{\tau}\right)$ & $\frac{1}{2 \sqrt{28}} \times 7$ & $\frac{1}{2}$ & -1 & 0 & 0 & -1 & 0 & 0 & 0 \\
\hline$(e, \mu, \tau)$ & $-\frac{1}{2 \sqrt{28}}$ & $-\frac{1}{2}$ & -1 & 0 & 0 & -1 & -1 & -2 & 0 \\
\hline
\end{tabular}

magnitude, the fact that all fermions but the neutrino have the same $\lambda^{63}$ tells us that at the Planck scale all of the $e, u, d$ have the same mass, and that the differences among these masses that we detect at observable energies stems from the differences introduced by the other vertical generators $I_{L}^{3}, B-L, Q$. So we now see that collectively speaking, the three generators $\lambda^{63}, \lambda^{48}, \lambda^{35}$ are all responsible for providing the mass degrees of freedom to the fermions, with $\lambda^{63}$ providing a vertical freedom to differentiate among $v, e, u, d$, and with $\lambda^{48}, \lambda^{35}$ providing two more horizontal degrees of freedom to differentiate the mass spectrum for a given fermion type into three permitted generational values. To the extent that one regards the quantum degrees of freedom that lead to discrete fermion masses as related to gravitational interactions given that mass and gravitation are inextricably linked, we now conclude that all three of $\lambda^{63}, \lambda^{48}, \lambda^{35}$ are the quantum generators of gravitational interactions, similarly to how $\lambda^{8}, \lambda^{3}$ generate strong interactions. But these $\lambda^{63}, \lambda^{48}, \lambda^{35}$ act differently from $\lambda^{8}, \lambda^{3}$ insofar as 1) $\lambda^{63}$ acts vertically while $\lambda^{48}, \lambda^{35}$ act horizontally, and 2) $\lambda^{48}, \lambda^{35}$ only start to act horizontally after they decouple from the other vertical generators at $v_{G U T}$ as a consequence of the lepto-quark symmetry breaking using the vacuum $\Phi=v_{G U T}(B-L)$. This is examined further in Table 5 below.

Finally, what this tells us is that in order to ascertain an answer to the question "why do the fermions have the masses they have?", the theoretical answer is this: follow the $\lambda^{63}, \lambda^{48}, \lambda^{35}$ generators, understand how $\lambda^{48}, \lambda^{35}$ separate out and start to act horizontally at $v_{P}$ and $v_{G U T}$, and understand how the masses evolve as one moves downward in energy from there toward the masses we do observe in the laboratory. In this regard, if $\lambda^{63}$ is used to break symmetry at or near the Planck scale as in (5.1), then we immediately see a break via $S U(8) \rightarrow S U(7)$ $\times U(1)$ with the neutrino fractured from all the other fermions. So, we already lose one vertical generator, which we take to be $\lambda^{48}$, which decouples and becomes horizontal. Thus, below the Planck scale but above the GUT scale, we would expect to see two fermion generations. Then, as we pass downward through the GUT scale and break the lepto-quark symmetry as in (5.2), we drop down to $S U(6)_{B} \times S U(2)_{L}$ and now two of the generators have decoupled from vertical to horizontal giving rise to a third generation. It would therefore make sense to believe that the observed substantial variation from first to second generation masses, and then again from second to third generation, has it origin in this sequential breaking of symmetry that starts with one generation at the Planck scale, turns into two generations between the Planck scale and the GUT lepto-quark scale, and turns into three generations below the GUT scale. At each scale as one "cools down," the masses become "frozen" in a manner of thinking. And it would seem to make sense due to their relatively larger masses that the high mass fermions, namely the $v_{\tau}, \tau, t, b$, are the ones that already exist in precursor form at the Planck scale, that the $v_{\mu}, \mu, c, s$ arise between the Planck scale and the 
Table 5. Mass degrees of freedom afforded by the gravitational interaction, below GUT energy.

\begin{tabular}{|c|c|c|c|}
\hline & $\lambda^{\prime 48}=\frac{1}{\sqrt{3}}, \lambda^{135}=0$ & $\lambda^{\prime 48}=-\frac{1}{2 \sqrt{3}}, \lambda^{\prime 35}=\frac{1}{2}$ & $\lambda^{\prime 48}=-\frac{1}{2 \sqrt{3}}, \lambda^{135}=-\frac{1}{2}$ \\
\hline$G=-\frac{1}{2 \sqrt{28}} \times 7$ & $v_{e}$ & $v_{\mu}$ & $v_{\tau}$ \\
\hline$G=\frac{1}{2 \sqrt{28}}$ & $u_{R}$ & $c_{R}$ & $t_{R}$ \\
\hline$G=\frac{1}{2 \sqrt{28}}$ & $d_{G}$ & $s_{G}$ & $b_{G}$ \\
\hline$G=\frac{1}{2 \sqrt{28}}$ & $d_{B}$ & $s_{B}$ & $b_{B}$ \\
\hline$G=\frac{1}{2 \sqrt{28}}$ & $e$ & $\mu$ & $\tau$ \\
\hline$G=\frac{1}{2 \sqrt{28}}$ & $d_{R}$ & $s_{R}$ & $b_{R}$ \\
\hline$G=\frac{1}{2 \sqrt{28}}$ & $u_{G}$ & $c_{G}$ & $t_{G}$ \\
\hline$G=\frac{1}{2 \sqrt{28}}$ & $u_{B}$ & $c_{B}$ & $t_{B}$ \\
\hline
\end{tabular}

GUT scale, and that the $v_{e}, e, u, d$ which predominate and are the ground states at observable energies are the last generation to emerge, below the $\sim 10^{15} \mathrm{GeV}$ scale at which the lepto-quark symmetry is broken and the $\lambda^{48}, \lambda^{35}$ decouple from other generators. Perhaps what happens at each symmetry breaking stage is that the one (or two) generations which exist before symmetry breaking "spin off" a portion of their mass to make two (or three) fermions when the generators decouple. That is, for example, what is "one electron" above the Planck scale has to become "two electrons" below the Planck scale, and these then have to further turn into three electrons below the GUT scale, at the same time that the generators are decoupled.

One final point before concluding this section pertains to chiral symmetry. Because the left-chiral generator $Y_{L}=B-L$ for all fermions, at the same time that we break symmetry at the GUT energy using (5.2) and (5.3), we have also forced a breaking of chiral symmetry. That is, the weak interactions start to become chiral nonsymmetric at the GUT scale, as part and parcel of the $Y_{L}=B-L$ symmetry breaking. As discussed briefly at the end of Section 5 of [1], baryon and meson physics is endemically, organically non-chiral, which is consistent with what is experimentally observed, all with $\gamma^{5}=i \gamma^{0} \gamma^{1} \gamma^{2} \gamma^{3}$ being the mainspring. Via what may be thought of as Dirac's “quinternian" progression $\gamma^{5}=i \gamma^{0} \gamma^{1} \gamma^{2} \gamma^{3}$ from Hamilton's quaternion $i^{2}=j^{2}$ $=k^{2}=i j k=1$, any time one has what looks like a "vector" object from one viewpoint, one can use $\gamma^{5}=i \gamma^{0} \gamma^{1} \gamma^{2} \gamma^{3}$ to create an "axial" object from another "dual" viewpoint, and "vector" and "axial" turn out to have a duality relationship that is integral to the Dirac algebra, all using "duality" based on the work of Reinich [9] later elaborated by Wheeler [10] which uses the Levi-Civita formalism (see [11] at pages 87-89). So given the degree to which baryon physics is fundamentally non-chiral courtesy of a Dirac algebra for which $\gamma^{5}=i \gamma^{0} \gamma^{1} \gamma^{2} \gamma^{3}$ is as integral to fermion physics as $i j k=1$ is to spatial rotations, it makes perfect sense that as soon as protons and neutrons are crystalized into being as stable magnetic monopoles by $Y_{L}=B-L$ symmetry breaking, we also bring about the non-chiral nature of the weak and weak hypercharge interactions.

\section{The Geometrodynamic Planck Vacuum, and What Makes the Neutrino Different (or, Let's Finally Catch that Mischievous Neutrino)}

With all that we have learned in Section 5, let us make a second pass through the Planck scale, and to see what else we may be able to learn.

It has long been believed, and experimentally given credence by the Lamb-Retherford shift in electromagnetic phenomenon, that near the Planck length,

$1.61624 \times 10^{-35}$ meters, and over Planck time scales of $5.39121 \times 10^{-44} \mathrm{sec}$, there is a violent sea of vacuum perturbations near the Planck energy $1.221 \times 10^{19} \mathrm{GeV}$, see the earlier referenced $[7,8]$ where this is developed in detail. It is also well-understood that energy fluctuations 
of this magnitude on such a small scale do have the effect of topologically creating microscopic black holes, also called wormholes, with a Schwarzschild radius at or near the Planck length. Let us now take a closer look at exactly what is believed to occur at this scale. Again, along the lines discussed in Section 2, it is unlikely that humans will ever be able to directly observe physics at the Planck length, but the development of such physics in the context of a GUT may lead us to low energy mass and energy predictions which - if they accord with empirical data - could then give us some confidence that the GUT which leads to such accord is also describing the Planck-length physics "behind the veil" with some semblance of accuracy.

When Wheeler talks in his seminal work [8] about the geometrodynamic Planck vacuum, the vacuum he envisions is constructed from a series of simple algebraic calculations with which it is important to be familiar. So let us review those here. First, Newton's law of gravitation $F=-G m_{1} m_{2} / r^{2}$ contains a numerator $G m_{1} m_{2}$ which has the same dimensions as the natural constant $\hbar c$. So the Planck mass $M_{P}^{2}$ is defined as the unique, natural mass unit formed out of the Newtonian numerator from $G, \hbar$ and $c$, namely:

$$
G M_{P}^{2} \equiv \hbar c .
$$

The above means that $M_{P}=\sqrt{\hbar c / G}$ so that the Planck energy $E_{P}=M_{P} c^{2}=\sqrt{\hbar c^{5} / G}$. The Fermi vev energy $v_{F}$ is similarly defined using the Fermi constant via $\sqrt{2} G_{F} v_{F}^{2} / c^{4} \equiv \hbar c$, with the $\sqrt{2}$ having historical origins based on how $G_{F}$ was first defined before electroweak interactions were well-understood. Comparing "apples to apples" the correspondence is $G \leftrightarrow \sqrt{2} G_{F}$.

The reduced Compton wavelength of a Planck mass (6.1) is easily calculated to be:

$$
\lambda_{P}=\hbar / M_{P} c=\sqrt{\frac{G \hbar}{c^{3}}} .
$$

Now we consider a large collection of Planck masses $M_{P}$ separated from one another by $\lambda_{P}$, in what would be a natural state of resonance. The negative gravitational potential energy $E_{G}$ between any two $M_{P}$ separated by $\lambda_{P}$ is easily calculated to be:

$$
E_{G}=-\frac{G M_{P}^{2}}{\lambda_{P}}=-\frac{\hbar c}{\lambda_{P}}=-\sqrt{\frac{\hbar c^{5}}{G}}=-E_{P} .
$$

But this is simply the negative of the Planck energy! So as Wheeler first surmised, a collection of Planck mass fluctuations (on average) separated by the Planck length (on average) averages out to be a vacuum because the negative gravitational energy precisely cancels the positive Planck energies which are posited in the first place, on average. Nonetheless, in very localized regions on the order of $\lambda_{P}$, there are very violent fluctuations of very high energy occurring. This is the so-called "geometrodynamic vacuum."

It is also important to note that the Schwarzschild "black hole" radius for a (non-rotating) Planck mass may be calculated to be:

$$
r_{S}=\frac{2 G M_{P}}{c^{2}}=\frac{2 G}{c^{2}} \sqrt{\frac{\hbar c}{G}}=2 \sqrt{\frac{G \hbar}{c^{3}}}=2 \lambda_{P} .
$$

Because the black hole radius is twice as large as the Planck length, this means that all of these fluctuations are occurring out of sight, behind a black hole horizon.

On top of this, Hawking [12] teaches seventeen years after Wheeler's initial elaboration of the geometrodynamic vacuum, based on general relativistic gravitational theory, that black holes emit a blackbody radiation spectrum. So if we recognize that the Planck vacuum is a vacuum in which the masses on average are Planck masses separated on average by the Planck length, and then like any good student of statistics we ask the natural follow up question "what is the actual statistical distribution of these energies about the average?" Hawking provides a clear answer: because these fluctuations are occurring behind an event horizon, the distribution is observed externally to the event horizon as a thermodynamic, blackbody spectrum. It would also make sense, therefore, to consider the prospect that when we observe blackbody radiation in the natural world, we are in fact observing a gravitational phenomenon from the Planck vacuum screened through over twenty orders of magnitude, which would render the blackbody spectrum that kicked off the quantum revolution in 1901 [13], a conesquence of gravitational theory. So much for disunion between gravitational theory and quantum theory!

But returning to GUTs, the Wheeler vacuum also teaches us something about the generator $\lambda^{63}$ with

$$
\operatorname{diag}\left(\lambda^{63}\right)=\frac{1}{2 \sqrt{28}} \times(7,-1,-1,-1,-1,-1,-1,-1)
$$

which we are associating on a preliminary basis with gravitation, which is this: One may look at the Planck vacuum in one of two entirely equivalent ways: First, one can say that there are a tremendous number of fluctuations with positive energy $+E_{P}$ on average, separated by $\lambda_{P}$ on average, thus giving rise to an equal amount of negative gravitational energies $-E_{P}$ on average, thus resulting in a vacuum on average, which has a gravitational blackbody distribution of energy when viewed from outside the event horizon, and which is redshifted as our observational perch recedes to that from which Planck first characterized this distribution. Second, one can start with negative energy fluctuations, separate them by $\lambda_{P}$, and they will gravitate to produce positive energy fluctuations. Each way of looking at this is equally 
valid. It is a "chicken and the egg" question. One can develop an equally sensible description of the exact same physics no matter where one starts: positive Planck masses producing negative gravitational energies, or negative Planck masses producing positive gravitational energies. It does not matter. These are two alternative descriptions of exactly the same thing.

Now, let's talk about specific fermions, such as the $\left(v, u_{R}, d_{G}, d_{B}, e, d_{R}, u_{G}, u_{B}\right)$ of our $\mathrm{SU}(8)$ GUT group. How do these actually take root in the vacuum? How are they "conceived" and "born"? Through the lens of 1957, referring to electromagnetic charge $Q$, Wheeler says in [8] that "classical charge appears as the flux of lines of force trapped in a multiply connected metric ... trapped by the topology of the space." In other words, charge gets "trapped" in the black hole wormholes. Updating this with all that we have learned in the intervening half century especially about Yang-Mills gauge theories and how charges such as the electric charge arise from the generators of Yang-Mills theory, we might say that these Planck-mass fluctuations "trap" the Yang-Mills internal symmetries (which include the electric charge), and that this is how particles are "born." Or, in parlance we introduce here, the physical fermions $\left(v, u_{R}, d_{G}, d_{B}, e, d_{R}\right.$, $\left.u_{G}, u_{B}\right)$ arise when a Planck-scale fluctuation is "fertileized" by the Yang-Mills generators of internal symmetry. So a neutrino $v$ is conceived when a fluctuation with Planck mass magnitude is fertilized by the generator eigenvalues in Table 2 corresponding to the neutrino. The same holds true for the up quark (in three colors), the down quark (in three colors) and the electron. Then, as Wheeler points out, the particles we observe from 20 orders of magnitude lower, have had all but the most miniscule portion of their original $\sim M_{P}$ masses cancelled/averaged out by the positive and negative energy fluctuations of the vacuum, leaving behind only a small mass residue which results from the trapping of the field lines, i.e., from the fertilization. Those are the particles and masses we observe.

But if the Planck vacuum raises a chicken and the egg question, the next question is this: how does nature decide whether the egg comes first or the chicken comes first? Does nature fertilize the positive energy fluctuations into observed particles, or the negative energy ones? Or, might she fertilize both? And what would a fertilized positive energy fluctuation look like, versus a fertilized negative energy fluctuation? And, fundamentally, how is this precisely-balanced positive versus negative energy symmetry in the Planck vacuum broken, in favor of the very miniscule (relative to the Planck vacuum) preponderance of positive energy over negative energy that we observe in the material universe?

Now our

$$
\operatorname{diag}\left(\lambda^{63}\right)=\frac{1}{2 \sqrt{28}} \times(7,-1,-1,-1,-1,-1,-1,-1)
$$

generator provides the critical clue: If this is a gravitational generator as we have begun to surmise, and if this generator is actually used to break symmetry at or near the Planck energy as in (5.1), and given that this is the energy at which gravitation is dominant as is clear from (6.1) through (6.4), then this generator will have a great deal to do with how the Planck vacuum first gets fertileized to produce what we observe. So the gravitational charge of the neutrino being of opposite sign from the gravitational charges of all the other fermions suggests that perhaps neutrinos are fertilized negative energy Planck vacuum fluctuations and the up and down quarks and the electron are all fertilized positive energy Planck vacuum fluctuations. Not only would this neatly resolve the chicken and egg problem, but it would explain many other things as well, especially about the ever-elusive neutrino.

First, this would truly place neutrinos in a class by themselves. They would be born of negative energy Planck scale fluctuations, brought about via the gravitational interactions of positive energy Planck scale fluctuations. Other fermions are rooted in "Planck matter"; neutrinos are rooted in "Planck gravitation." Second, above the Planck energy, behind the event horizon, we would expect there to be a complete symmetry among all of the octuplet members $\left(v, u_{R}, d_{G}, d_{B}, e, d_{R}, u_{G}, u_{B}\right)$. Any one fermion can readily decay into any other, and all would exist in equal numbers as part of an octuplet set. Thus, any time there is a neutrino, there are also seven other fermions to go along with that neutrino. Then, after we break the symmetry and the neutrino hooks up with negative energy fluctuations and the other seven fermions hook up with positive energy fluctuations, we would have a seven-to-one ratio of fermions which are rooted in positive energy fluctuations over fermions rooted in negative energy fluctuations. So as we reached lower and lower energies, there would be a net dominance of positive energy-rooted fermions over negative energy-rooted fermions. As such, this could help to explain how the positive versus negative energy symmetry of the Planck vacuum becomes broken. This is especially so given the fact that at low energies the neutrino masses become so very much smaller than all the other fermion masses.

Third, while we conventionally hold to the view that all matter gravitates the same way as all other matter, this would tell us that this conventional wisdom holds true for all matter except the neutrino. Below the Planck scale, the neutrino would fundamentally be a fermion rooted in negative energy fluctuations, while all of the other fermions would be rooted in positive energy fluctuations. This could certainly provide some degree of confidence that as we start to trace the development of the fermions from the Planck scale down to the laboratory scale, we 
may come to understand why $m_{u} / m_{e}=4.351853369$ and $m_{d} / m_{e}=9.601723351$, while $m_{e} / m_{v}>250,000$. The neutrino would start off in the Planck vacuum with a negative energy $\sim-M_{P}+\varepsilon_{v}$ where $\varepsilon_{v}$ represents the alteration in energy due to the fertilization of the negative energy gravitational fluctuation, while all the other fermions $f$ would start off with a positive energy $\sim+M_{P}+\varepsilon_{f}$ rooted in the matter fluctuations. Then, after screening of twenty orders of magnitude, the neutrino mass would end up very close to, and slightly larger than zero, and the rest of the fermion masses would end up more substantially above zero, with the observed masses between $2.5 \times 10^{5}$ and $2.5 \times 10^{6}$ times as large as what is observed for the neutrino.

Further, if the neutrino gravitates differently from every other fermion (which we shall explore even further in the next section), then its elusive, idiosyncratic behaveiors may be much better understood. From a technology viewpoint, this also suggests that if one ever hopes to develop technologies to "shield" gravitation or overcome gravitational attraction other than by the brute force of rocket propulsion, the neutrino would be central to that undertaking. Harvesting and controlling the elusive neutrino, however, would be the core technology challenge. And, since neutrinos do exist throughout the universe as elusive as they may be, this would also mean that cosmological theories based on the supposition that all matter gravitates in relation to all other matter in exactly the same way would have to be modified to recognize that the neutrino defies this supposition.

As a consequence of the forgoing, let us now choose a negative gravitational charge for the neutrino to go with the negative energy fluctuations, as a matter of convention. Then, let us introduce the hypothesis - which needs to be borne out through detailed calculation of its conesquences - that the neutrinos are in fact conceived at or near the Planck scale when negative energy gravitational fluctuations in the Planck vacuum become fertilized with the negative gravitational charge of the neutrino

$v=\left|-\lambda^{63}=-\frac{1}{2 \sqrt{28}} \times 7\right\rangle$, and that quarks and electrons are born at or near the Planck scale when positive energy gravitational fluctuations in the Planck vacuum become fertilized with the positive gravitational charge of a quark or an electron $u, d, e=\left|-\lambda^{63}=+\frac{1}{2 \sqrt{28}}\right\rangle$.

And in this regard, choosing the convention of a negative gravitational charge for the neutrino to go with the negative Planck energy fluctuations, we now explicitly define a gravitational interaction generator:

$$
G \equiv-\lambda^{63} ; \operatorname{diag}(G)=\frac{1}{2 \sqrt{28}} \times(-7,1,1,1,1,1,1,1) .
$$

We may find occasion to adjust this coefficient $\frac{1}{2 \sqrt{28}}$ as we calculate from this point forward, but this sign reversal, and the identification of $\lambda^{63}$ with a gravitational generator $G$, makes clear 1) that the neutrino is understood to gravitate differently than all the other fermions as we shall further examine in a moment, and 2) that the neutrino is rooted in negative energy Planck fluctuations while all the other fermions are rooted in positive fluctuations. Or, as Wheeler might say, the neutrino lines of force are trapped in negative energy topological wormholes, and the quark and electron lines of force are trapped in positive energy topological wormholes.

\section{Spontaneous Symmetry Breaking, Fermion and Generator Fractures, and Intergenerational Cabibbo Mixing of Left-Chiral Hypercharge Doublets}

As we now return to spontaneous symmetry breaking, it will be important to develop an understanding of what we shall call "fermion fractures" and "generator fractures." While the fermion fracturing we are about to describe may already be implicitly understood as a feature a spontaneous symmetry breaking, it is important to make this understanding explicit, as this will play a crucial role in understanding generation replication, and especially, the Cabibbo mixing which for leptons leads to so-called neutrino oscillations (which have been largely responseble for demonstrating that the neutrino does have some tiny mass, contrary to what may have been believed two or three decades ago).

When a gauge group has not been broken at all, and assuming that fermions have been assigned to the fundamental representation of that gauge group, then any one fermion is completely free to decay into any other fermion. $\mathrm{SU}(3)_{\mathrm{QCD}}$ provides a good example of this. As we can see from Table 1, or as will be understood in any event, there are three color eigenstates

$$
\begin{aligned}
& R=\left|\lambda^{8}=\frac{1}{\sqrt{3}}, \lambda^{3}=0\right\rangle, G=\left|\lambda^{8}=-\frac{1}{2 \sqrt{3}}, \lambda^{3}=\frac{1}{2}\right\rangle, \\
& B=\left|\lambda^{8}=-\frac{1}{2 \sqrt{3}}, \lambda^{3}=-\frac{1}{2}\right\rangle .
\end{aligned}
$$

The symmetry is not broken, so any of these eigenstates may freely decay into any other one of these eigenstates, even though their quantum numbers are different. For example, all three color states $R, G, B$ have completely different $\lambda^{3}$, namely, $\lambda^{3}=0, \frac{1}{2},-\frac{1}{2}$, yet they freely transition among themselves, which is central to QCD interactions. Similarly, as just discussed, above the Planck scale any fermion may transition into any 
other fermion.

Once a symmetry is broken, however, some fermions become "fractured" from some other fermions, and they are forbidden from decaying into one another except under very limited conditions. It is these limited conditions which are of central interest in the discussion following.

Let us first break the symmetry of $\mathrm{SU}(8)$ at the Planck scale using (5.1), which we recast in light of (6.5) as:

$$
\begin{aligned}
& \Phi_{P} \cong v_{P} G, \text { i.e. }, \\
& \operatorname{diag}\left(\Phi_{P}\right)=\operatorname{diag}\left(\lambda^{i} \varphi_{P i}\right) \\
& \cong \frac{1}{2 \sqrt{28}} v_{P}(-7,1,1,1,1,1,1,1)
\end{aligned}
$$

What then happens? Of course, similarly to what was discussed in Section 8 of [1], the vacuum commutes such that $\left[\Phi_{P}, \lambda^{i}\right]=0, i=1, \cdots, 48$.

It also self-commutes with $G$, that is, $\left[\Phi_{P}, G\right]=v_{P}[G, G]=0$.

But our real interest here is to look at the fermions themselves.

The neutrino, with $v=\left|G=-\frac{1}{2 \sqrt{28}} \times 7\right\rangle$, becomes fractured from all the other fermions with $\left|G=\frac{1}{2 \sqrt{28}}\right\rangle$, and can no longer decay into any of these other states via the generator $G$ that was used to break the symmetry. It would be as if the red quarks in QCD were suddenly forbidden from decaying into green or blue quarks - but of course they can do so because the QCD symmetry is never broken. If $G$ is a gravitational generator, then the neutrino can no longer undergo a gravitational decay through $G$ into any other fermion. What does that mean? The neutrino will no longer gravitate with any other fermion except for another neutrino! But - and this is critical-it may still undergo other types of decay through the generators of other interactions. Let's elaborate:

If the neutrino is to decay into any other fermion after the symmetry is broken via (7.1), it must decay into a fermion via an interaction governed by an interaction generator other than $\lambda^{63}$ gravitation such that the fermion has the same charge value under that other interaction generator as that of the neutrino. Referring to Table 2 to make this clear, this means that the neutrino still can undergo a $\lambda^{35}$ decay into a $u_{R}$ quark because each has $\lambda^{35}=0$. And it can still undergo a $I_{L}^{3}$ decay into any up quark, because these and the neutrino all have $I_{L}^{3}=\frac{1}{2}$. Most importantly, as will become central in the discussion be- low, the neutrino can still undergo $B-L=Y_{L}$ decay into an electron because both the neutrino and the electron have the same $B-L=Y_{L}=-1$ and so form a doublet under $B-L=Y_{L}$. This latter ability for the neutrino and the electron to decay into one another as like-charge members of a $B-L=Y_{L}=-1$ doublet, lasts until the electroweak symmetry is finally broken at much lower (Fermi vev) energies into the electromagnetic interaction.

Now let's look at the remaining seven fermions. Even after the symmetry breaking (7.1), these fermions are completely free to decay into one another via the gravitational generator $G$, because they are all like-valued $\left|G=\frac{1}{2 \sqrt{28}}\right\rangle$ eigenstates of $G$. They all continue to gravitate with one another, while the neutrino steps aside and stops gravitating with them. Indeed, starting at the Planck scale, and until one drops down to GUT energies on the order of $10^{15} \mathrm{GeV}$, these seven other fermions remain part of an SU(7) septuplet. Since all of these fermions are united by the common characteristic that they are born through the fertilization of positive $(+)$ energy vacuum fluctuations, we shall refer to this group as SU(7)+. Thus, between the Planck scale and the GUT scale, the gauge group is $S U(7)_{+} \times U(1)_{G}$, and the topologically-stable $\mathrm{SU}(7)$ magnetic monopoles with all the fermions of $\mathrm{a}^{2} \mathrm{H}$ atom are

$\pi_{1}\left(S U(7)_{+}\right) \times \pi_{1}\left(U(1)_{G}\right)=\pi_{1}\left(U(1)_{G}\right)=\mathbb{Z}$. The $U(1)_{G}$ emanates from the commutation of $\left[\Phi_{P}, G\right]=v_{P}[G, G]=0$, and is based on a neutrino singlet $v=\left|G=-\frac{1}{2 \sqrt{28}} \times 7\right\rangle$ plus a septuplet of the remaining fermions all of which are in $\left|G=\frac{1}{2 \sqrt{28}}\right\rangle$ states. Again, importantly, the neutrino can no longer interact gravitationally via $\lambda^{63}$ with any of the remaining seven fermions. If it is to interact with them, it must do so via other non-gravitational interactions.

Now let's progress down the energy scale and break symmetry with $B-L=Y_{L}$ in the vicinity of $v_{G U T} \sim 10^{15} \mathrm{GeV}$ using (5.2). The residual gauge groups are now those shown and discussed in (5.6), but let's again look closely at how the fermions are fractured, and let's also look at the loss of two generators going from $S U(8) \rightarrow S U(6)$.

Referring now to Table 4, the $\lambda^{48}, \lambda^{35}$ generators are no longer in play as vertical generators, because two generators are lost going from $\mathrm{SU}(8)$ to $\mathrm{SU}(6)$. These do not disappear entirely, but become horizontal as already discussed, in a manner we shall momentarily develop further. As to the remaining five linearly-independent vertical generators in Table 4, the electrons and the quarks still remain a gravitational septuplet and so can still interact gravitationally with one another (while the neutrino does not)! Following the rule that after symmetry 
breaking the only decays which are permitted are decays under a given generator for which the decaying fermions have a like-charge, the remaining decays options are as among members of the quark sextuplet of fermions with $B-L=\frac{1}{3}$, and between the lepton doublet of fermions with $B-L=-1$. The former decays among fermions in the $B-L=\frac{1}{3}$ sextuplet, consist of QCD strong interacttions decays among the $R, G, B$ color eigenstates based on the $\lambda^{\prime 8}, \lambda^{\prime 3}$ generators, and weak decays between states with $\left|Y_{L}=\frac{1}{3}, I_{L}^{3}= \pm \frac{1}{2}\right\rangle$ due to the common $Y_{L}=\frac{1}{3}$. The latter decays between the two fermions in the $B-L=-1$ lepton doublet, consist of weak decays between the neutrino and the electron with $\left|Y_{L}=-1, I_{L}^{3}= \pm \frac{1}{2}\right\rangle$ due to the common $Y_{L}=-1$.

Now, however, most importantly, the quarks have become fully fractured from the leptons, and there is no more decay permitted between quarks and leptons. This is because, referring to Table 4, there is not a single vertical generator other than $\lambda^{63}$ for which any quark shares the same charge as any lepton, so hereafter, the only way for a quark to interact with a lepton is gravitationally. And the neutrino - the odd man out - does not interact gravitationally with any other fermions besides another neutrino, because its gravitational charge is different from that of all the other fermions and that gravitational generator was used to break the Planck symmetry.

Further, as was developed in detail in Section 8 of [1], the breaking of $B-L$ also creates stable magnetic monopoles $\pi_{1}\left(S U(3)_{C^{\prime}}\right) \times \pi_{1}\left(U(1)_{B}\right)=\pi_{1}\left(U(1)_{B}\right)=\mathbb{Z}$ which manifest as protons and neutrons forming $(p, n)$ doublets with $B=1$. So this is also the symmetry break at which protons and neutrons are born. And, with $B-L=Y_{L}$, as noted at the end of Section 5, the weak interaction becomes non-chiral to go along the with chiral non-symmetry of baryon interactions as discussed in Section 5 of [1].

So the $B-L$ symmetry breaking is responsible for several interrelated phenomena: it brings about the three generations observed at low energy, it brings about protons and neutrons, it forecloses lepto-quark decays, and because $B-L=Y_{L}$, it brings about the broken chiral symmetry of the weak interactions.

Now, at some level, everything discussed so far in this section about fermion fracturing due to symmetry breaking restates what is likely obvious, because it is known that one of the very basic consequences of symmetry breaking is that it forecloses certain decays which are permitted to occur in the higher state of symmetry before the symmetry is broken. From a thermodynamic view, it "freezes out" certain transitions below a certain critical temperature (recognizing too that some symmetries are not broken but are actually restored on the opposite end of the scale, near absolute zero, where electrons are superconducted freely without any apparent friction from the protons and neutrons from which they separate at GUT energies, which suggests that superconductivity may well be a phenomenon at which the SU(7) symmetry between electrons and quarks is restored so electrons can flow through rather than around protons and neutrons). But the reason for focusing on fermion fracturing in this way, is because we will now venture into the not-obvious realm of generation replication and apply these observations to understand what happens there as well.

If the rule is that after symmetry breaking fermions can only decay into other fermions with like-charges under some interaction that was not used to break the symmetry, then what happens to the horizontal generators $\lambda^{48}, \lambda^{35}$ after $B-L=Y_{L}$ symmetry breaking? Not only have quarks become fractured from leptons, but $\lambda^{48}, \lambda^{35}$ have themselves become fractured from the other generators! So we not only have fermion fracturing, we have generator fracturing. If we follow suit, then it would seem that a similar set of rules may well apply. Let's explore.

First, referring to Table 2,

$$
\operatorname{diag}\left(\lambda^{48}\right)=\frac{1}{2 \sqrt{21}}(0,6,-1,-1,-1,-1,-1,-1)
$$

and

$$
\operatorname{diag}\left(\lambda^{35}\right)=\frac{1}{2 \sqrt{15}}(0,0,5,-1,-1,-1,-1,-1)
$$

are the two fractured generators. Because these no longer differentiate an observable vertical symmetry, but still do provide two degrees of freedom as illustrated in Figure 1 in section 5, let us transform these two generators into $\lambda^{\prime 48}, \lambda^{\prime 35}$ with the eigenvalues shown in Figure 1. No new calculation is required: we simply use (4.3) and (4.4) but without $\lambda^{8}$ and $\lambda^{3}$, and so redefine $\lambda^{48}, \lambda^{35} \rightarrow$ $\lambda^{\prime 48}, \lambda^{\prime 35}$ according to:

$$
\begin{aligned}
\lambda^{\prime 48} & \equiv \frac{\sqrt{7}}{3} \lambda^{48}-\frac{2}{3 \sqrt{5}} \lambda^{35}-\sqrt{\frac{2}{15}} \lambda^{24}, \\
\lambda^{\prime 35} & \equiv \sqrt{\frac{3}{5}} \lambda^{35}-\sqrt{\frac{2}{5}} \lambda^{24} .
\end{aligned}
$$

It is readily seen that

and

$$
\operatorname{diag}\left(\lambda^{\prime 48}\right)=\frac{1}{2 \sqrt{3}}(0,2,-1,-1,0,0,0,0)
$$

$$
\operatorname{diag}\left(\lambda^{\prime 35}\right)=\frac{1}{2}(0,0,1,-1,0,0,0,0)
$$


So these generators now do yield the SU(3) configuretion shown in Figure 1, albeit with eight eigenstates, five of which are all zero-valued and trivial, and three of which are not. We can now label these three non-trivial eigenstates as:

$$
\begin{aligned}
& e \equiv\left|\lambda^{\prime 48}=\frac{1}{\sqrt{3}}, \lambda^{\prime 35}=0\right\rangle, \\
& \mu \equiv\left|\lambda^{\prime 48}=-\frac{1}{2 \sqrt{3}}, \lambda^{\prime 35}=\frac{1}{2}\right\rangle, \\
& \tau \equiv\left|\lambda^{\prime 48}=-\frac{1}{2 \sqrt{3}}, \lambda^{\prime 35}=-\frac{1}{2}\right\rangle,
\end{aligned}
$$

just as illustrated in Figure 1. However, these are now free-floating generators once the $B-L=Y_{L}$ symmetry is broken, so they no longer provide vertical symmetry quantum numbers for any of the fermions, as illustrated in Tables 3 and 4. Rather, they appear to provide a replication of each fermion into three generations. But if this is the case, then they should lead to other facets of generation replication as well, including Cabibbo-type mixing, and to the observation that the only way a particle from one generation can transform into a particle of another generation is via left-chiral weak interaction decays from one weak isospin to a different weak isospin, and not directly. As we shall now see, this is a consequence of the fermion and generator fracturing highlighted above and the "freezing" restrictions that come into play after symmetry breaking.

Because the generators $\lambda^{48}, \lambda^{35}$ have become fractured from the other generators, and given what we know about the fermion generations from experimental observations, it appears that each of the $e, \mu, \tau$ eigenstates is fractured from one another so that it is now forbidden for a direct transition to take place between any of the three states (7.4), (7.5), (7.6), i.e., no decays may take place any longer via the $\lambda^{\prime 48}, \lambda^{\prime 35}$ (or $\lambda^{48}, \lambda^{35}$ ) interaction generators. Symbolically, $e \leftrightarrow \mu \leftrightarrow \tau$. Any decays that do take place, must occur via another generator for which the charges are the same as among the fermions involved in the decay. The fermion has to find a "loophole." This is exactly like the discussion we had at the beginning of this section about the neutrino in relation to the remaining fermions from which it becomes fractured at $v_{P}$, or the fracturing of the quarks from the leptons at $v_{G U T}$. In order to undergo decay into a different fermion, a fermion must find a different generator and a different fermion which has the same charge as the original fermion with respect to that different generator.

So referring to Table 4, if a first-generation $e$ fermion is to decay into a second generation $\mu$ fermion or a third-generation $\tau$ fermion, it must to do so via a generator other than $\lambda^{\prime 48}, \lambda^{\prime 35}$, into a fermion for which it shares an identical charge for that other generator. For the leptons, this is straightforward: the electron and the neutrino share a common charge $B-L=Y_{L}=-1$, and so for a first generation electron to become a second generation electron, it must go from $e \rightarrow v_{\mu} \rightarrow \mu$ or $e \rightarrow v_{e} \rightarrow \mu$, all of which have the same $B-L=Y_{L}$ $=-1$. This is the only remaining "decay loophole." Again this is exactly what was discussed earlier with regard to fermion fracturing. And so, for the first time, we see Cabibbo mixing and neutrino oscillations, because that is exactly how these work as well. This also explains flavor non-conservation as regards the generations: at the end of $e \rightarrow v_{\mu} \rightarrow \mu$ or $e \rightarrow v_{e} \rightarrow \mu$, what started as a first generation electron is now a second generation electron and neither $\lambda^{\prime 48}$ nor $\lambda^{\prime 35}$ is conserved, and this is because the generators are fractured.

For the quarks it is a little more complicated, because this transition rule needs to be strengthened due to strong interactions. In particular, if a fermion can undergo a $e \leftrightarrow \mu \leftrightarrow \tau$ transition by decay through at least one generator that is the same for both, then, for example, referring to Table 4, one could observe a $u_{R} \leftrightarrow c_{G}$ transition, because both the $u_{R}$ and the $c_{G}$ have the same $B-L=Y_{L}=\frac{1}{3}$. This would imply that Cabibbo mixing can occur not only via weak but also via strong interactions, and the latter, of course, is not observed.

So for horizontal symmetry transitions, it appears that we have to tighten the rules even further. Specifically, it appears that for a horizontal transition to be permitted, not just one, but all of the vertical degrees of freedom in Tables 3 and $\mathbf{4}$ must be the same as between the two fermions involved in the decay. Table 3 actually illustrates this rule the best, because this rule says that a horizontal $e \leftrightarrow \mu \leftrightarrow \tau$ transition must occur either as a transition between the first and fifth, second and sixth, third and seventh, or fourth and eighth fermions in Table 3. These are the fermion doublets which share a common:

$$
\begin{aligned}
& (v, e) \equiv\left|B-L=-1, \lambda^{\prime 8}=0, \lambda^{\prime 3}=0, Y_{L}=-1\right\rangle, \\
& \left(u_{R}, d_{R}\right) \equiv\left|B-L=\frac{1}{3}, \lambda^{\prime 8}=\frac{1}{\sqrt{3}}, \lambda^{\prime 3}=0, Y_{L}=\frac{1}{3}\right\rangle, \\
& \left(u_{G}, d_{G}\right) \\
& \equiv\left|B-L=\frac{1}{3}, \lambda^{\prime 8}=-\frac{1}{2 \sqrt{3}}, \lambda^{\prime 3}=\frac{1}{2}, Y_{L}=\frac{1}{3}\right\rangle, \\
& \left(u_{B}, d_{B}\right) \\
& \equiv\left|B-L=\frac{1}{3}, \lambda^{\prime 8}=-\frac{1}{2 \sqrt{3}}, \lambda^{\prime 3}=-\frac{1}{2}, Y_{L}=\frac{1}{3}\right\rangle
\end{aligned}
$$

So in sum, one can have neither $e \leftrightarrow \mu \leftrightarrow \tau$, nor $v_{e} \leftrightarrow v_{\mu} \leftrightarrow v_{\tau}$, nor $u \leftrightarrow c \leftrightarrow t$, nor $d \leftrightarrow s \leftrightarrow b$ tran- 
sitions, because each of these has different $\lambda^{\prime 48}, \lambda^{\prime 35}$ eigenvalues. These states are all fractured from one another. One cannot have intergenerational transitions between $(v, e)$ and any of the quark doublets because these have been fractured from one another by $B-L$ breaking. One cannot have intergenerational $R \leftrightarrow G$ $\leftrightarrow B$ transitions among (7.8), (7.9) and (7.10) because although QCD is never broken, the QCD generators are different as among red, green and blue states. If any vertical generators, or any horizontal generators are different as between two fermions, then based on what we observe, the apparent rule is that the horizontal transition is not permitted. So all that is permitted - the only "loophole" left for decay—are the $v \leftrightarrow e, u_{R} \leftrightarrow d_{R}, u_{G}$ $\leftrightarrow d_{G}$ and $u_{B} \leftrightarrow d_{B}$ transitions, because these are the only transitions for which all of the generators listed are the same for both fermions. And here, because of the tightened rules when it comes to horizontal transitions based on fractured generators, even the right-chiral generator $Y_{R}$ is excluded, because this too is not the same as between the members of each of the above doublets. This is why we show $Y_{L}$ in the above but not $Y_{R}$. This means only the left-chiral states may participate in transitions among the $e \leftrightarrow \mu \leftrightarrow \tau$ states in (7.4) to (7.6). Observationally, we know that this is also a characteristic of left-chiral weak generational interactions.

These stronger rules for the horizontal generators may at first seem arbitrary, but they are not. They may be understood because for the horizontal generators, not only are some fermions fractured from other fermions, but the horizontal generators themselves are fractured from the vertical generators. It is the fracturing of both generators and fermions which leads to such stringency. So for a vertical generator that breaks symmetry but is not itself fractured from the other vertical generators, transitions are permitted so long as at least one other vertical generator provides the same charge as between the two transition states. But for a generator which has itself been fractured from the other generators, the rule is even more restrictive. Now, transitions are permitted only if all of the involved vertical generators provide the same charge as between the two transition states.

Now, the astute reader may notice that the electric charge $Q$ and left-chiral weak isospin $I_{L}^{3}$ are also not the same as between the two fermions in any of the doublets in (7.7) through (7.10) above. $Q(v, e)=(0,-1)$ and $Q(u, d)=\left(\frac{2}{3},-\frac{1}{3}\right)$ as between the members of these doublets, as well as $I_{L}^{3}(v, e)=\left(\frac{1}{2},-\frac{1}{2}\right)$ and $I_{L}^{3}(u, d)=\left(\frac{1}{2},-\frac{1}{2}\right)$. And so, the question might be asked, why are even these interactions permitted? After all, this changes the generators also, so by these rules, shouldn't this be forbidden also? But further reflection makes this answer clear: the electric charge does not emerge as a physically-preclusive generator until it is used to break the electroweak symmetry at much lower energies determined by the Fermi vacuum $v_{F}=$

$246.219651 \mathrm{GeV}$. This is the same way in which $B-L$ is not a preclusive generator until its breaks symmetry at GUT energies. So indeed, once we break electroweak symmetry, no transitions are permitted between generations. But at the same time, neiter will $v \leftrightarrow e$ or $u \leftrightarrow d$ be permitted, but this is because weak interacttions are no longer permitted either (in the historical sense that the weak interaction becomes "weak"). So what we learn from this, is that the ability of fermions to change generations will wax and wane in lock step with the weak interaction itself and the breaking of electroweak symmetry, just as is observed!

By imposing the more stringent rule that once the $\lambda^{48}, \lambda^{35}$ interaction generators have become fractured from the other generators by $B-L$ symmetry breaking at $v_{G U T} \sim 10^{15} \mathrm{GeV}$, no horizontal transitions are permitted among the (7.4) to (7.6) states unless all of the remaining vertical generators - chiral symmetric or notare the same as between the fermions involved in the transition, we arrive at precisely the type of mixing that is observed in nature as among the three generations. This makes generation mixing part and parcel of weak interactions, while excluding the strong interactions and even the right-chiral states from participation in generational mixing.

So, now we take the final, formal steps to mathematically represent all of these decay restrictions. Referring to Section 12.12 of [14], the two generators $\lambda^{\prime 48}, \lambda^{\prime 35}$ introduce two degrees of freedom and so define threenon-trivial horizontal eigenstates $e, \mu, \tau$ in (7.4) through (7.6) and Figure 1, representing eigenstates of SU(3), which states are precluded from direct transformation into one another according to the rules just outlined because they are fractured generators. $\mathrm{SU}(3)$ can be used to form unitary matrices $U$ with $9=3 \times 3$ components. Because the only permitted transitions are (7.7) through (7.10), we can alter the phase of any of the $2 \times 3=6$ quark states which we designate $(u \therefore, d \therefore)$

$=(u, c, t, d, s, b)$ following Table 3, without altering the physics. Similarly for leptons. But one may omit an overall phase change which still leaves the physics invariant. This means that $U$ must be a function of $9=3 \times 3$ minus $6=2 \times 3$ plus 1 parameters, i.e., 4 parameters. But an orthogonal $3 \times 3$ matrix only has $C(3,2)=3$ real parameters, which leaves one residual phase. So for the leptons $l$, we may choose to form this matrix in the representation: 


$$
U_{l}=\left(\begin{array}{ccc}
1 & 0 & 0 \\
0 & c_{2} & s_{2} \\
0 & -s_{2} & c_{2}
\end{array}\right)\left(\begin{array}{ccc}
c_{1} & s_{1} & 0 \\
-s_{1} & c_{1} & 0 \\
0 & 0 & \mathrm{e}^{i \delta}
\end{array}\right)\left(\begin{array}{ccc}
1 & 0 & 0 \\
0 & c_{3} & s_{3} \\
0 & -s_{3} & c_{3}
\end{array}\right)_{l}=\left(\begin{array}{ccc}
c_{1} & s_{1} c_{3} & s_{1} s_{3} \\
-s_{1} c_{2} & c_{1} c_{2} c_{3}-s_{2} s_{3} \mathrm{e}^{i \delta} & c_{1} c_{2} s_{3}+s_{2} c_{3} \mathrm{e}^{i \delta} \\
s_{1} s_{2} & -c_{1} s_{2} c_{3}-c_{2} s_{3} \mathrm{e}^{i \delta} & -c_{1} s_{2} s_{3}+c_{2} c_{3} \mathrm{e}^{i \delta}
\end{array}\right)_{l}
$$

and for the quarks $q$ we form the analogous:

$$
U_{q}=\left(\begin{array}{ccc}
1 & 0 & 0 \\
0 & c_{2} & s_{2} \\
0 & -s_{2} & c_{2}
\end{array}\right)\left(\begin{array}{ccc}
c_{1} & s_{1} & 0 \\
-s_{1} & c_{1} & 0 \\
0 & 0 & \mathrm{e}^{i \delta}
\end{array}\right)\left(\begin{array}{ccc}
1 & 0 & 0 \\
0 & c_{3} & s_{3} \\
0 & -s_{3} & c_{3}
\end{array}\right)_{q}=\left(\begin{array}{ccc}
c_{1} & s_{1} c_{3} & s_{1} s_{3} \\
-s_{1} c_{2} & c_{1} c_{2} c_{3}-s_{2} s_{3} \mathrm{e}^{i \delta} & c_{1} c_{2} s_{3}+s_{2} c_{3} \mathrm{e}^{i \delta} \\
s_{1} s_{2} & -c_{1} s_{2} c_{3}-c_{2} s_{3} \mathrm{e}^{i \delta} & -c_{1} s_{2} s_{3}+c_{2} c_{3} \mathrm{e}^{i \delta}
\end{array}\right)_{q}
$$

To implement the lepton mixing, we keep in mind from (7.7) that for a $e \leftrightarrow \mu \leftrightarrow \tau$ transition to take place which alters the quantum numbers in (7.4) through (7.6), we cannot go directly from $e \leftrightarrow \mu \leftrightarrow \tau$, but must engage in a vertical transition between the states $(v, e)$ in which all of the generators $B-L=-1, \lambda^{\prime 8}=0$, $\lambda^{\prime 3}=0, Y_{L}=-1$ do not change. The only permitted tran-

sition is $v \leftrightarrow e$. Now, one can always apply (7.11) to both of $(v, e)$, but then one of them can always be transformed into a pure state while the other is similarly transformed, without changing the physics. In other words, all that is observable is the relative transition as between $(v, e)$. So following the usual conventions, we use (7.11) to transform the lower members of the $(v, e)$ doublet, that is, we define:

$$
e_{i}^{\prime}=\left(\begin{array}{c}
e^{\prime} \\
\mu^{\prime} \\
\tau^{\prime}
\end{array}\right) \equiv\left(\begin{array}{ccc}
c_{1} & s_{1} c_{3} & s_{1} s_{3} \\
-s_{1} c_{2} & c_{1} c_{2} c_{3}-s_{2} s_{3} \mathrm{e}^{i \delta} & c_{1} c_{2} s_{3}+s_{2} c_{3} \mathrm{e}^{i \delta} \\
s_{1} s_{2} & -c_{1} s_{2} c_{3}-c_{2} s_{3} \mathrm{e}^{i \delta} & -c_{1} s_{2} s_{3}+c_{2} c_{3} \mathrm{e}^{i \delta}
\end{array}\right)_{l}\left(\begin{array}{l}
e \\
\mu \\
\tau
\end{array}\right)=U_{l} e_{i}
$$

Similarly for the quarks of each color $C=R, G, B$, we define:

$$
d_{C}^{\prime}=\left(\begin{array}{c}
d_{C}^{\prime} \\
s_{C}^{\prime} \\
b_{C}^{\prime}
\end{array}\right) \equiv\left(\begin{array}{ccc}
c_{1} & s_{1} c_{3} & s_{1} s_{3} \\
-s_{1} c_{2} & c_{1} c_{2} c_{3}-s_{2} s_{3} \mathrm{e}^{i \delta} & c_{1} c_{2} s_{3}+s_{2} c_{3} \mathrm{e}^{i \delta} \\
s_{1} s_{2} & -c_{1} s_{2} c_{3}-c_{2} s_{3} \mathrm{e}^{i \delta} & -c_{1} s_{2} s_{3}+c_{2} c_{3} \mathrm{e}^{i \delta}
\end{array}\right)_{q}\left(\begin{array}{l}
d_{C} \\
s_{C} \\
b_{C}
\end{array}\right)=U_{q} d_{C i}
$$

Because $Y_{R}$ is not the same as between the members of each of the (7.7) through (7.10) doublets, right-chiral transitions are also precluded, and the only permitted transitions are for left-chiral states. So these will be projected with $\frac{1}{2}\left(1-\gamma^{5}\right)$. Further, because $\lambda^{\prime 8}, \lambda^{\prime 3}$ are not the same except as between members of the four distinct doublets in (7.7) through (7.10), the only permitted transitions will be between one lepton and another lepton, and between a first quark of a given color and a second quark of the same color $C=R, G, B$. This keeps the strong QCD interaction out of generation-changing transitions (and also out of any CP violation), and makes this an exclusively weak, left-handed chiral phenomenon. So for leptons, the transition currents will be:

$$
\begin{aligned}
J_{l}^{\mu} & =\left(\begin{array}{lll}
v_{e} & v_{\mu} & v_{\tau}
\end{array}\right) \gamma^{\mu}\left(\begin{array}{ccc}
c_{1} & s_{1} c_{3} & s_{1} s_{3} \\
-s_{1} c_{2} & c_{1} c_{2} c_{3}-s_{2} s_{3} \mathrm{e}^{i \delta} & c_{1} c_{2} s_{3}+s_{2} c_{3} \mathrm{e}^{i \delta} \\
s_{1} s_{2} & -c_{1} s_{2} c_{3}-c_{2} s_{3} \mathrm{e}^{i \delta} & -c_{1} s_{2} s_{3}+c_{2} c_{3} \mathrm{e}^{i \delta}
\end{array}\right) \frac{1}{2}\left(1-\gamma^{5}\right)\left(\begin{array}{l}
e \\
\mu \\
\tau
\end{array}\right) \\
& =v_{i} \gamma^{\mu} U_{l} \frac{1}{2}\left(1-\gamma^{5}\right) e_{i}=v_{i} \gamma^{\mu} \frac{1}{2}\left(1-\gamma^{5}\right) e_{i}^{\prime}=v_{i L} \gamma^{\mu} e_{i L}^{\prime}
\end{aligned}
$$

And for quarks of each color $C=R, G, B$, they will be:

$$
\begin{aligned}
J_{q}^{\mu} & =\left(\begin{array}{lll}
u_{C} & c_{C} & t_{C}
\end{array}\right) \gamma^{\mu}\left(\begin{array}{ccc}
c_{1} & s_{1} c_{3} & s_{1} s_{3} \\
-s_{1} c_{2} & c_{1} c_{2} c_{3}-s_{2} s_{3} \mathrm{e}^{i \delta} & c_{1} c_{2} s_{3}+s_{2} c_{3} \mathrm{e}^{i \delta} \\
s_{1} s_{2} & -c_{1} s_{2} c_{3}-c_{2} s_{3} \mathrm{e}^{i \delta} & -c_{1} s_{2} s_{3}+c_{2} c_{3} \mathrm{e}^{i \delta}
\end{array}\right)_{q} \frac{1}{2}\left(1-\gamma^{5}\right)\left(\begin{array}{c}
d_{C} \\
s_{C} \\
b_{C}
\end{array}\right) \\
& =u_{i C} \gamma^{\mu} U_{q} \frac{1}{2}\left(1-\gamma^{5}\right) d_{i C}=u_{i C} \gamma^{\mu} \frac{1}{2}\left(1-\gamma^{5}\right) d_{i C}^{\prime}=u_{i L C} \gamma^{\mu} d_{i L C}^{\prime}
\end{aligned}
$$


This is exactly what the phenomenology demonstrates!

So, returning to the question posed at the very outset of the discussion following Table 2, not only does SU(8) not provide too much freedom, but upon careful consideration and development, it provides exactly the right amount of freedom to explain the precisely observed fermion phenomenology of three generations. Further, by applying the rule that fermions which are fractured from one another after symmetry breaking cannot decay into one another except by a vertical interaction other than the vertical interaction that was used to break symmetry, and that decay with regards to a fractured generator which thereafter becomes a free-floating horizontal degree of freedom is only permitted between fermion eigenstates for which all of the surviving vertical generators are the same, we can use $\mathrm{SU}(8)$ to explain everything that we know about the qualitative features of the interactions we observe, from generation replication to weak chiral non-symmetry to Cabibbo mixing to the fact that this mixing occurs only via weak isospin decays between left-handed states. And in the process we have perhaps found that neutrinos do not gravitate with any fermions aside from other neutrinos, which is likely to be of tremendous consequence as this is better developed and understood and especially if it can ever be exploited.

Before concluding this section, let us now return to the first three generators $\lambda^{63}, \lambda^{48}, \lambda^{35}$ of SU(8). Based on the earlier review of how $\lambda^{63}$ breaks symmetry near the gravitational Planck scale and sets the neutrino on a trajectory to have a mass orders of magnitude smaller than that of any other fermion; given how the $\lambda^{48}, \lambda^{35}$ fracture from the other vertical generators and form the basis for two horizontal degrees of freedom that underlie three fermion generations in which one fermion is distinguished from one another solely by mass and not by any other quantum numbers from a vertical degree of freedom, and given that mass and gravitation are inextricably linked such that gravitation is the "mass interaction," we now formally associate these three generators $\lambda^{63}, \lambda^{48}$, $\lambda^{35}$ with the gravitational interaction, at the elementary particle level, below the GUT energy. Using (7.4) to (7.6) and (6.5), we highlight this connection in Table 5 of Section 5 .

The horizontal degrees of freedom from $\lambda^{48}, \lambda^{35}$ which to enable the fermions in each generation to have distinct masses in relation to their counterparts in the other two generations are shown horizontally, while the vertical degree of freedom $G$ enabling each fermion within a generation to have a distinct mass is shown vertically. Of course, with $\mathrm{SU}(3)_{C}$ remaining unbroken, different colors of the same flavor of quark within one generation have the same mass. As noted earlier, using the $\therefore$ notation, the vertical gravitational generator $G$ does not distinguish the $u \therefore, d \therefore, e \therefore$ masses from one an- other within a generation. So at high energies, as noted, the fermions (other than neutrinos) within a generation all have the same mass. It is only through the stages of symmetry breaking and the remaining generators $B-L=Y_{L}, I_{L}^{3}$ and $Q$, that the mass spectrum within a generation separates. This may be thought of as mass/ energy differences emanating from strong, weak, and electromagnetic interactions, i.e., one may regard quark masses to differ from electron masses because they are quarks not leptons, and up and down quark masses to differ because their weak isospins and electric charges are different. Gravitational generators provide the freedom for these differences to occur.

As to interactions, after all symmetry breaking includeing electroweak symmetry breaking is completed, the seven generators of SU(3) now are allocated as follows: three degrees of freedom go to gravitation in the form of $\lambda^{63}, \lambda^{\prime 48}, \lambda^{\prime 35}$, two degrees of freedom go to strong QCD interactions via $\lambda^{18}, \lambda^{\prime 3}$, one degree of freedom goes to left-chiral weak interactions via $I_{L}^{3}$, and the final degree of freedom goes to electromagnetic interactions via $Q$. Seven linearly-independent degrees of freedom, and eight vertical fermion eigenstates, thus account perfectly, with nothing missing and nothing superfluous, for the observed phenomenology of the fermions and their interactions, including generation replication and Cabibbo mixing, left-chiral weak interactions, and the elusive and perhaps gravitationally-defiant behavior of the neutrino.

\section{Summary and Conclusion}

We have in the foregoing focused on the breaking of symmetry at the Planck scale and the GUT scale, which, astronomical observation aside, is many orders of magnitude beyond what we may ever hope to observe directly. The final stage of symmetry breaking is electroweak symmetry breaking at the Fermi vev $v_{F}=$ $246.219651 \mathrm{GeV}$. This is in the realm of observation, and the generator used to break this symmetry is the electric charge generator $Q$. This final symmetry break gives rise to the electromagnetic interaction which dominates atomic and chemical structure and much of what is most directly observed in the natural world beyond gravitational interactions. That is, beyond objects falling to earth and planets wandering the heavens along prescribed trajectories, electromagnetic phenomena in electromagnetic and chemical and atomic form are our first line of direct experience of the natural world. Our experience of nuclear phenomena-based on the protons and neutrons which come to life as stable magnetic monopoles at the GUT scale as has been reviewed here and in [1] - comes to us through the laboratory instrumentation that we used to extend the range of our physi- 
cal senses, and gives rise to the vast preponderance of the matter that populates and animates the universe.

When we break the electroweak symmetry we make use of the electric charge generator (4.6), and analogously to (5.1) through (5.3), employ the Fermi vacuum:

$$
\Phi_{F}=\lambda^{i} \varphi_{i F}=v_{F} Q,
$$

which specifically means that:

$$
\begin{aligned}
& \operatorname{diag}\left(\Phi_{F}\right)=\operatorname{diag}\left(T^{i} \varphi_{i F}\right) \\
& \equiv v_{F}\left(0, \frac{2}{3},-\frac{1}{3},-\frac{1}{3},-1,-\frac{1}{3}, \frac{2}{3}, \frac{2}{3}\right)=v_{F} \operatorname{diag} Q
\end{aligned}
$$

Picking off the coefficients from the generators in (4.6), for each non-zero component of the vacuum we then have:

$$
\begin{aligned}
& \varphi_{F}^{48}=\frac{2}{3} \sqrt{\frac{7}{3}} v_{F} ; \varphi_{F}^{35}=-\frac{4}{3 \sqrt{15}} v_{F} ; \varphi_{F}^{24}=-\frac{2}{3} \sqrt{\frac{2}{5}} v_{F} ; \\
& \varphi_{F}^{15}=-2 \sqrt{\frac{2}{3}} v_{F} ; \varphi_{F}^{8}=-\frac{2}{\sqrt{3}} v_{F}
\end{aligned}
$$

which leads to:

$$
\begin{aligned}
& \left(\varphi_{F}^{48}\right)^{2}+\left(\varphi_{F}^{35}\right)^{2}+\left(\varphi_{F}^{24}\right)^{2}+\left(\varphi_{F}^{15}\right)^{2}+\left(\varphi_{F}^{8}\right)^{2} \\
& =\left(\frac{4 \times 7}{9 \times 3}+\frac{16}{9 \times 15}+\frac{4 \times 2}{9 \times 5}+\frac{8}{3}+\frac{4}{3}\right) v_{F}^{2} \\
& =\frac{16}{3} v_{F}^{2}=C^{2} v_{F}^{2}
\end{aligned}
$$

and consequently an electroweak Clebsch-Gordon coefficient:

$$
C=\frac{4}{\sqrt{3}} .
$$

This is how the electroweak symmetry is broken for the $\mathrm{SU}(8)$ group that we have developed throughout this paper. This final symmetry break fractures all fermions of different electric charges from one another, and so precludes their decay into one another. Referring to Table 4, weak isospin transitions between up and down quarks with differing charges $Q(u, d)=\left(\frac{2}{3},-\frac{1}{3}\right)$ are now precluded, as are similar transitions between electrons and neutrinos with $Q(v, e)=(0,-1)$. This shuts down the weak interaction (in the historical view, renders it "weak"; in hindsight it is probably better called the "faint" interaction), and because weak isospin decays as reviewed in the last section are the only avenues permitted for generation-changing transitions, generational transitions also are turned off in lock step. The only transitions still permitted after electroweak symmetry breaking, given that $Q$ is a vertical symmetry generator and so not subject to the very stringent rules laid out in the last section for horizontal transitions, are the vertical, colorchanging $R, G, B$ transitions of QCD, which are still allowed to occur because the quarks involved in these interactions are part of a triplet in which $B-L=\frac{1}{3}$ is the same for each, and the QCD symmetry remains unbroken. That is, the only permitted decays once electroweak symmetry is broken, are decays along the $B-L$ generator for particles of like $B-L$ with unbroken $\lambda^{\prime 8}, \lambda^{\prime 3}$ generators, which, of course, are strong QCD interactions. With the exception of the $R \leftrightarrow G$ $\leftrightarrow B$ transitions of QCD, no fermion may transform into any other different type of fermion.

Now, following three stages of symmetry breaking - at the Planck scale, the GUT scale and the Fermi scale-all of the fermions have become fractured from one another, generation transitions cease, and the particles are frozen into the configurations of our everyday experience. The SU(8) symmetry with seven generator degrees of freedom that we started with in Table 2 still does exist, but it has become hidden and distorted behind twenty orders of magnitude of vacuum screening and three stages of symmetry breaking that have fractured neutrinos from the other fermions and broken off their gravitational communication, broken the Planck symmetry between positive and negative energy fluctuations, fractured quarks from leptons, fractured two generators from the remaining five to provide horizontal generational replication, brought about Cabibbo-type mixing among these generations for left-handed chiral projections only, and finally, fractured the upper and lower members of the like-hypercharge $Y_{L}$ (weak isospin) doublets from one another, turned off the weak interactions, and frozen the particles in place so that all we observe at the lowest energies are electromagnetic and strong interactions, as well as the bulk interaction of gravitating masses which is eluded by the neutrino.

This GUT, which is based on the hypothesis that baryons are Yang-Mills magnetic monopoles and is rooted in the $\mathrm{SU}(4)_{\mathrm{P}}$ and $\mathrm{SU}(4)_{\mathrm{N}}$ subgroups developed in Section 7 of [1] which yielded over half a dozen accurate predicttions in [1,2] as reviewed in Section 1 here, leads systematically to all of the qualitative particle and interaction phenomenology which we are able to observe with our senses and the extension of our senses through experimental apparatus. But the confirmation of the particular GUT proposed here, versus other possible GUTs which reproduce similar phenomenally, needs to come through mass and energy predictions which continue the successful empirical matches developed in $[1,2]$. As discussed in Section 3, one would expect that these energy predictions should come about by developing the remaining $\Phi$-containing terms in the Lagrangian density (3.2) which we have not yet developed, and then making 
use of these to calculate various energies $E=-\iiint \mathfrak{L d}^{3} x$ to be matched up with empirical data. Along the way, the development should proceed on a parallel course to that of Sections 2 through 11 of [1], making use of the non-Abelian Klein-Gordon Equation (3.10), representing scalar sources as $J \equiv \bar{\psi} \psi$, employing the same sort spin sums and the same Gaussian ansatz modeling of fermions that was developed respectively in Sections 3 and 9 of [1], and keeping in mind the clues we have elaborated in (3.6) through (3.8) and (3.11) here, all while employing the GUT and symmetry breaking that has been elaborated here.

It is clear from $[1,2]$ that it will be possible via this approach to calculate and predict definitive mass and energy values, just as has been done previously in [1] and [2]. It will then be left to interpret those values as we did in Sections 11 and 12 of [1] and throughout [2], and to compare them with experimental data to try to ascertain the meaning of those calculations and predictions to obtain sensible numerical matches to observed energy data. That is, we clearly will be able to calculate energies. The question will be whether the energies we are able to calculate will match and make sense in relation to the empirical data as well as they did in $[1,2]$.

Success in this endeavor, if it should arrive, would validate that this particular GUT may indeed be the one that nature has selected to govern the phenomenology of the material universe, and would provide some confidence that the development elaborated here does reach "behind the veil" to explain how nature really does operate in energy domains likely to forever remain beyond the reach of our direct senses and the extension of our senses gained through experimental devices and methods.

\section{REFERENCES}

[1] J. R. Yablon, "Why Baryons Are Yang-Mills Magnetic Monopoles," Hadronic Journal, Vol. 35, No. 4, 2012, pp. 401-468.
http://www.hadronicpress.com/issues/HJ/VOL35/HJ-35-4 .pdf

[2] J. R. Yablon, "Predicting the Binding Energies of the 1s Nuclides with High Precision, Based on Baryons which are Yang-Mills Magnetic Monopoles," Journal of Modern Physics, Vol. 4 No. 4A, 2013, pp. 70-93. doi:10.4236/jmp.2013.44A010

[3] H. Georgi and S. Glashow, "Unity of All ElementaryParticle Forces," Physical Review Letters, Vol. 32, 1974, p. 438. doi:10.1103/PhysRevLett.32.438

[4] http://5dstm.org/

[5] G. E. Volovok, "The Universe in a Helium Droplet," Clarendon Press, Oxford, 2003.

[6] J. Beringer, et al., (Particle Data Group), "PR D86, $010001, " 2012$.

http://pdg.lbl.gov/2012/listings/rpp2012-list-neutrino-pro p.pdf

[7] C. W. Misner, K. S. Thorne and J. A. Wheeler, "Gravitation," Freeman, New York, 1973, p. 1190.

[8] J. A. Wheeler, "On the Nature of Quantum Geometrodynamics," Annals of Physics, Vol. 2, 1957, pp. 604-614. doi:10.1016/0003-4916(57)90050-7

[9] G. Y. Reinich, "Electrodynamics in the General Relativity Theory," Transactions of the American Mathematical Society, Vol. 27, No. 1, 1925, pp. 106-136. doi:10.1090/S0002-9947-1925-1501302-6

[10] J. A. Wheeler, "Geometrodynamics," Academic Press, Boston, 1962, pp. 225-253.

[11] C. W. Misner, K. S. Thorne and J. A. Wheeler, "Gravitation," W. H. Freeman \& Co., New York, 1973.

[12] S. W. Hawking, "Black Hole Explosions?" Nature, Vol. 248, No. 5443, 1974, pp. 30-31. doi:10.1038/248030a0

[13] M. Planck, "On the Law of Distribution of Energy in the Normal Spectrum," Annalen der Physik, Vol. 4, 1901, p. 553. doi:10.1002/andp.19013090310

[14] F. Halzen and A. D. Martin, "Quarks and Leptons: An Introductory Course in Modern Particle Physics," John Wiley \& Sons, Hoboken, 1984. 